\title{
Problematic of Drinking Water Access in Rural Area: Case Study of the Sourou Valley in Burkina Faso
}

\author{
Savadogo Boubacar ${ }^{1}$, Kaboré Aminata ${ }^{2}$, Zongo Dramane ${ }^{1}$, Poda Jean Noel ${ }^{1}$, Bado Hortense ${ }^{3}$, \\ Rosillon Francis $^{4}$, Dayeri Dianou ${ }^{1 *}$ \\ ${ }^{1}$ National Center for Scientific and Technological Researches, Institute for Health Sciences Research, Ouagadougou, Burkina Faso; \\ ${ }^{2}$ Research Center for Biological, Alimentary and Nutritional Sciences, Research and Training Unit, Life and Earth Sciences, Univer- \\ sity of Ouagadougou, Ouagadougou, Burkina Faso; ${ }^{3}$ Convention for the Promotion of a Sustainable Development, Non-Govern- \\ mental Organization, Ouagadougou, Burkina Faso; ${ }^{4}$ Water, Environment, Development Unit, Arlon Campus, University of Liège, \\ Arlon, Belgique. \\ Email: *dayerid@yahoo.fr
}

Received October $20^{\text {th }}, 2012$; revised November $17^{\text {th }}, 2012$; accepted December $15^{\text {th }}, 2012$

\begin{abstract}
Safe drinking water access for rural populations in developing countries remains a challenge for a sustainable development. The study aims to investigate the drinking water quality and the factors affecting this quality in the Sourou valley in Burkina Faso. A total of 135 water samples were collected in sterile glass bottles during the dry seasons 2007, 2008, and 2012 from 10 drillings and 5 wells. Fifteen physicochemical parameters and two fecal pollution indicators $(E s-$ cherichia coli and fecal Coliforms) were monitored based on laboratory standard methods. Datas were analyzed, using the Student's t-test and XLSTAT 7.5.2 statistical software. From results obtained, water quality was related to water source and sampling period as well $(\mathrm{p}<0.0001) .30 \%$ of drillings provided water with nitrates concentration over the World Health Organization (WHO) guideline value. High turbidity was also observed for some drillings. Moreover, $90 \%$ of drillings showed water total hardness largely over the WHO threshold value. Water from drillings were exempt of fecal pollution, contrasting with the wells one which appeared uniformly polluted with concentrations exceeding sometimes $10^{3}$ and $10^{4} \mathrm{CFU} / 100 \mathrm{ml}$ for $E$. coli and fecal Coliforms, respectively. Field investigations showed a preference of wells as drinking water source, and that appeared related to the lack of self-management of drillings and to cultural considerations. Overall, this study highlighted that a regular survey of water quality, management of protection zones around drinking water sources, sensitization on water resources self-management, hygiene and health issues, and providing appropriate household disinfection methods could help advancing to reach an effective safe drinking water access for rural populations in the country.
\end{abstract}

Keywords: Drinking Water; Chemistry; Bacteriology; Pollution; Sourou; Burkina Faso

\section{Introduction}

Access to safe drinking-water is important as a health and development issue at national, regional and local levels. In some regions, it has been shown that investments in water supply and sanitation can yield a net economic benefit, since the reductions in adverse health effects and health care costs outweigh the costs of undertaking the interventions. This is true for major water supply infrastructure investments through to water treatment in the home. Experience has also shown that intervenetions in improving access to safe water favor the poor in particular, whether in rural or urban areas, and can be an effective part of poverty alleviation strategies [1].

However, safe drinking water access for rural popula-

"Corresponding author. tions in developing countries (DC) remains a challenge to overcome for a sustainable development. Despite appreciable efforts undertaken to achieve the Millennium Development Goals (MDG), many of these countries are still suffering from a lack of drinking water access [2,3]. In view of the increase in water demand, measures undertaken generally focused the quantitative aspect to meet the needs of populations. However, beyond the quantitative aspect, it is advisable to pay attention on the quality of water consumed by the populations. Unfortunately, in most DC, analytical data of water quality are missing [4], although it is well known that the control of water in its different components is essential for the socioeconomic development of a country, and that also determine the implementation of the MDG in the other sectors $[5,6]$.

Considering water access for populations in Burkina 
Faso, the National Action Plan for Integrated Water Management Resources [7] depicted the country as in situation of hydric stress, the mean water resources available and accessible being $850 \mathrm{~m}^{3}$ per year and per inhabitant, compared to the threshold water shortage generally evaluated to $1000 \mathrm{~m}^{3}$. The situation is partially related to the fact that in most parts of the country, the aquifer system is located in a sedimentary zone with hard stones represented by sandstones and limestone-dolomites with a thickness estimated to a hundred meters $[8,9]$ and that limits strongly the possibility to realize drillings with a high flow and at low cost. As a consequence, the urban hydraulic resorts quite exclusively to surface waters to feet populations demand (i.e. dams of Ziga and Loumbila which actually aliment the capital of the country, Ouagadougou) [10].

At national level, the regulation on the quality of water resources is sustained by a decree which fixes the standards of pollutants in surface waters and drinking water as well with regards to WHO standards and the specific situations in the country [11]. Specifically for drinking water quality, the guideline values currently referred by the national office of water are the ones recommended by WHO [12].

According to the United Nations Development Program [13], the access to safe drinking water in Burkina Faso clearly improved these years with a national rate of water access passing from $18.3 \%$ in 1993 to $66.3 \%$ in 2007. These good performances are the consequence of the efforts undertaken by the country to achieve the Millennium Development Goals (MDG). These efforts led to the reinforcement of the infrastructures of water supply. The network of drinking water adduction which was of 881 kilometers in 1986 reached 3129 kilometers in 2004 while between 2006 and 2007, the projects and programs allowed the realization of approximately 1882 drillings [13]. However, the situation is undoubtedly variable from one place to another in the country, the urban environment being privileged compared to the rural one [14].

Although Burkina Faso already reached the MDG for the access to safe drinking water [13], the situation is not therefore satisfactory, in particular in rural environment where the populations are confronted with the optimal management of the water supply points. If in urban environment, water distributed is subjected to regular control, in rural area the indicators of drinking water quality are missing due to the lack of analytical data [15-17]. An improvement of knowledge is however essential to make the water services more efficient and to reinforce the policy for an effective access to safe drinking water in the country. From this view point, one of the recommendations of the National Action Plan for Integrated Water Management Resources (APIWRM), consists to the in- stallation of networks for water analysis $[7,18]$.

To meet this recommendation, a network for surface waters and groundwaters quality survey was initiated since 2006 within the framework of the Contract of the Sourou River [19]. Following a first investigation on the quality of surface waters [17], the present study examines the general physicochemical and bacterial parameters of water from wells and drillings consumed by populations in the Sourou valley.

After the presentation of the context of the study and the methodology used, the results obtained will be presented. The quality of water will be discussed with regard to the national and WHO standards [11,12,20-22], and the environmental factors and socio-behavioral attitudes impacting the access to safe drinking water before proposing some issues in order to improve the situation.

\section{Study Context}

As underlined in our previous works [15-17], the study was realized within the framework of the implementation of a project of river contract in the Sourou' watershed. In 2003, through cooperation with the Walloon Region of Belgium, a river contract based on the Walloon model was initiated [19]. This model is an approach of integrated and participatory management which aims at gathering within a river committee the representatives of all the users of water in order to define and implement a restoration actions program of the water resources, waterways and their accesses. This program was elaborated according to a consensual approach which takes care to integrate the concerns of each user while improving the protection of environment. In Burkina Faso, it was proved that the river contract could also be an issue to fight against desertification and poverty [19].

This project which has been developed on nearly ten years was framed locally by a NGO, the Convention for the promotion of a sustainable development (COPROD) which dealt with the animation and the coordination of the activities. The Department of Environment of the University of Liege in Belgium ensured the general coordination and the scientific expertise for the account of the Walloon Region in collaboration with the Institute for Health Sciences Research (IRSS) of the Scientific and Technological National Research Center (CNRST) of Ouagadougou.

Considering the operational characteristic of the river contract, many field activities were performed [23]. The activities were divided into five sets of themes: 1) Coordination, animation and dialog between actors of water; 2) Improvement of knowledge through the data acquisition and the organization of many formations for the users of water and the local collectivities; 3) Communication, 
information and sensitization of schools and water users; 4) Restoration of the water resources and the environment which is concretized by an improvement of water access and environment protection (waterways protection and fight against desertification); and 5) Income-generating activities in a context of fighting against poverty.

The present study is in line with an improvement of knowledge related to the quality of water resources in the Sourou basin. It also meets one of measures recommended by the APIWRM in the actions field No. 2 "water information system": action 2.2, the implementation of national networks to monitor the water quality, water uses, water requests and the risks [7].

\section{Study Zone}

The Sourou valley as previously described [15-17] is located in the North-West of Burkina Faso, in the area of the Mouhoun loop (Figure 1). The Sourou River takes its source in Mali at the level of Baye. It makes border between Burkina Faso and Mali, by then crossing the Burkina Faso from north to south before joining Mouhoun River at Léry. The Sourou' watershed covers a surface of $16,200 \mathrm{~km}^{2}$ but only the central part located on the left bank of the river (approximately $5000 \mathrm{~km}^{2}$ ) is the object of this study.

The Sourou valley is especially known for its hydroagricultural installations following the erection of dam valves at the junction of Sourou and Mouhoun rivers in 1984. The realization allowed to increase significantly the level of water of the Sourou River $\left(600,000,000 \mathrm{~m}^{3}\right)$ through the valley [15]. This availability of water thus allowed the creation of irrigated perimeters, making the Sourou valley an important agricultural production zone [15].

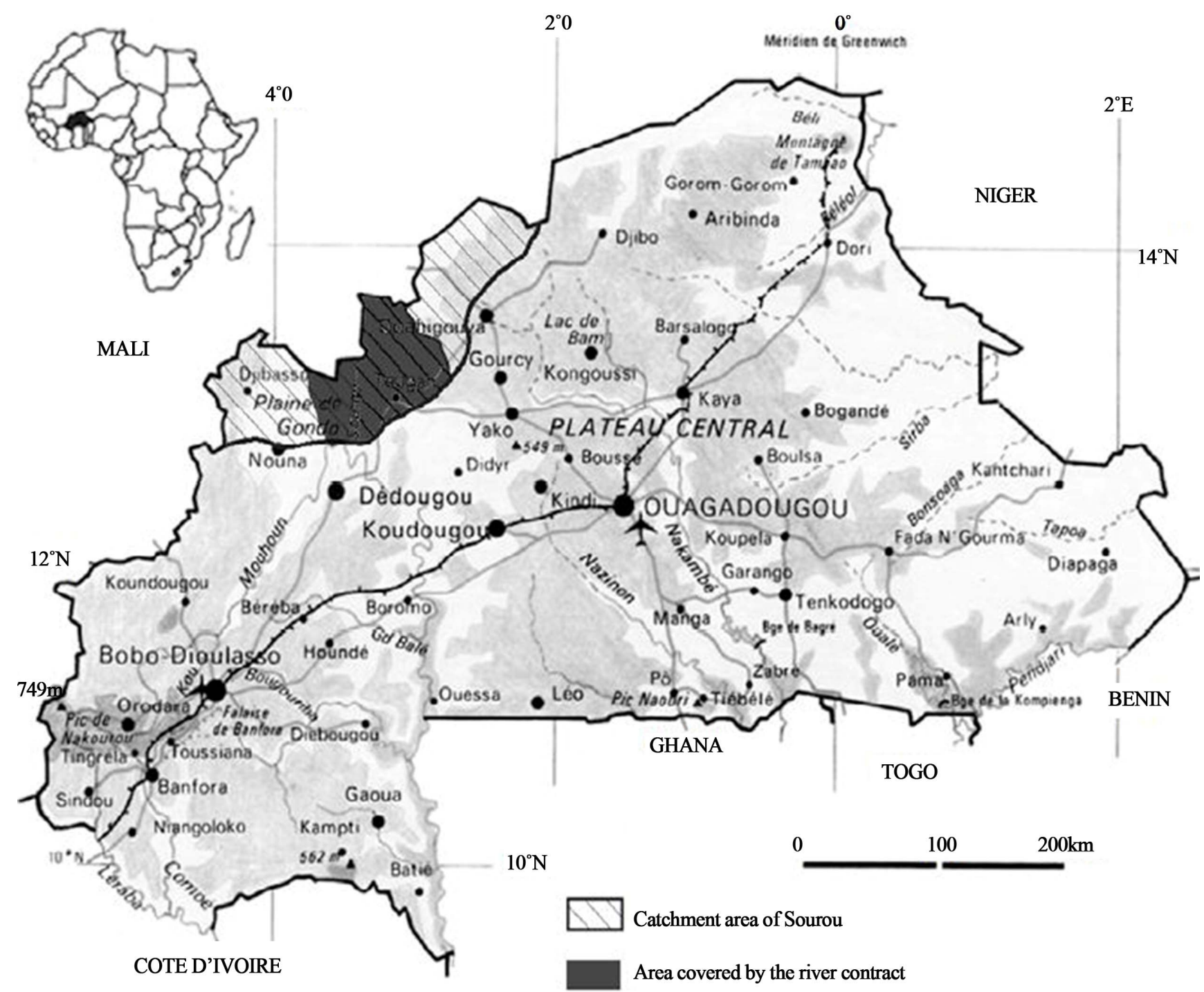

Figure 1. Map showing the catchment area of the Sourou valley and the zone covered by the river contract [19]. 
Currently, the irrigated perimeters extend on a surface of 3200 ha (which will be soon increased with a new additional zone of $2033 \mathrm{ha}$ ) and are managed under the direction of the Sourou Valley Development Authority (SVDA). The Sourou valley thus constitutes an important agrarian production zone benefitting the whole country [14].

Drinking water access for Sourou populations is possible from various sources of supply, mainly from groundwaters. Except for some more important localities like
Tougan, chief town of the Sourou Province, which profits from a partial system of water adduction, the rural populations generally feed on from traditional wells to a relatively dense network of drillings installed by NGO within the framework of cooperative projects or programs supported by the Government, notably the second Soils Management National Plan (SMNP 2); however, populations of insular villages or close to the Sourou river use surface water for their alimentation (Figure 2).
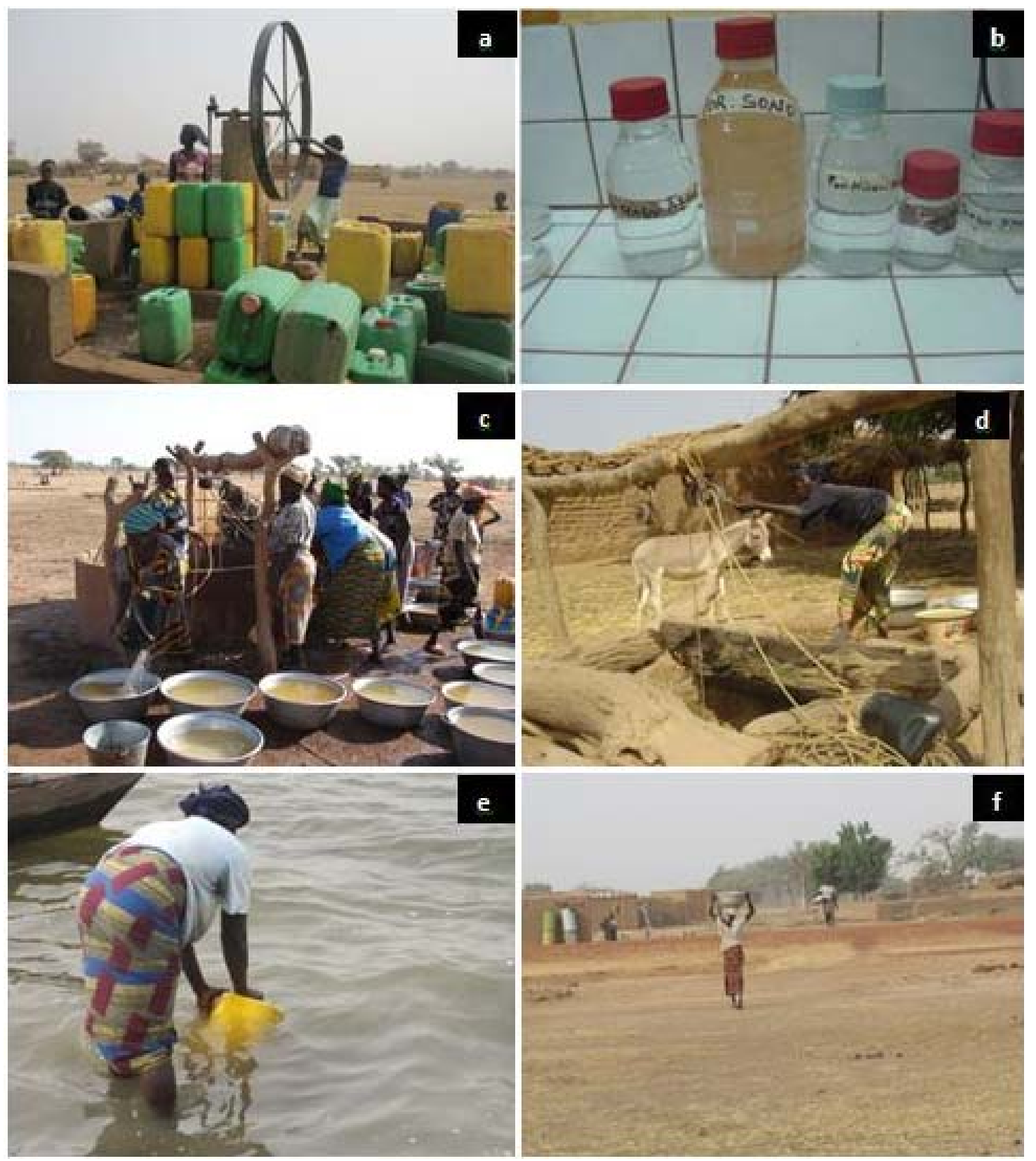

Figure 2. Some aspects of drinking water access in the Sourou valley. (a) Getting water from Niassan-AMVS drilling (January 2012); (b) Aspects of water sampled from drillings (November 2007); (c) Getting water from a modern well at Kouy (January 2012); (d) Getting water from the traditional well of Kouy-Mosque (March 2008); (e) Getting drinking water from Sourou River at Toma-island (January 2012); (f) Transportation of drinking water collected from drilling to home at Kiembara (January 2012). 
The availability of groundwater is rather stable. The Sourou aquifer system is located in a sedimentary zone and consists of hard stones represented by sandstones and limestone-dolomites which can be crossed by faults. The thickness of the aquifers' sandstones is estimated to a hundred meters [24]. The depth of drillings is about a sixty meters while the water level in the traditional wells is variable from one site to another, the depth being of approximately 10 to $20 \mathrm{~m}$ in the zone of the study. The refill of the aquifer can be established through a slow infiltration in the subsoil. This diffuse refill could be supplemented by a preferential water flow through fractured zones [14].

Within the framework of the Sourou river contract, an inventory of wells and drillings were carried out [19]. This inventory identified many non-functional works which were the object of repair within the framework of this river contract. More than 100 works were thus given back in activity.

\section{Material and Methods}

\subsection{Water Sampling and Field Information Gathering}

The groundwater resources focused in this study consisted of 10 drillings and 5 wells (traditional or modern, large diameter wells) located throughout the valley (Figure 3). Sampling campaigns were carried out in 2007, 2008 and 2012 during the dry season for which water demand and pressure on water sources were particularly high.

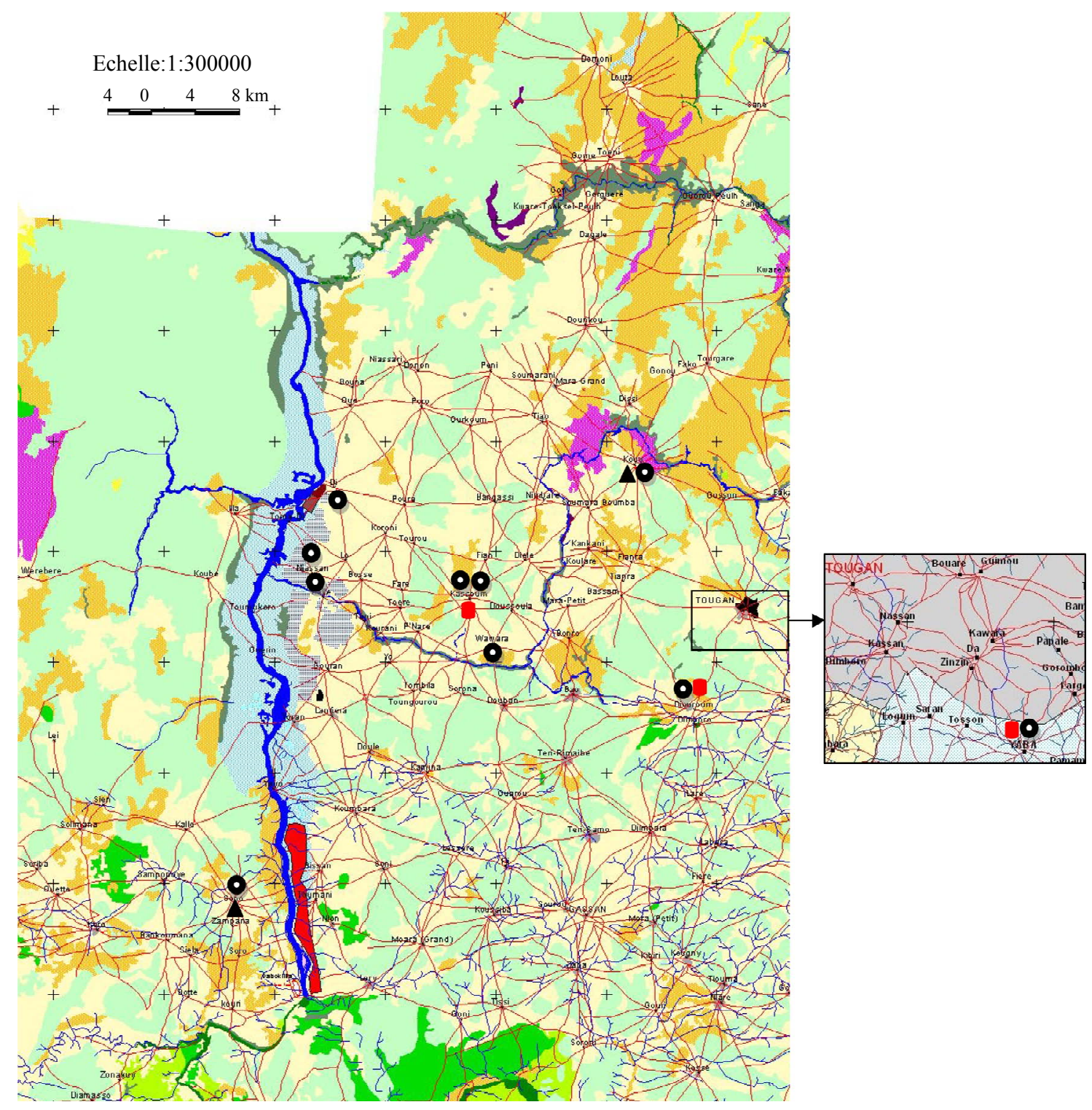

Figure 3. Location of sampling sources within the zone covered by the river contract in the Sourou valley [19]. Modern well (3); $\Delta$ Traditional well (2); $\odot$ Drilling (10). 
Water samples were collected aseptically in triplicate into sterile glass bottles at the field (Figure 2(b)). A total of 45 samples were taken and analyzed during each campaign. Samples collected at the field were preserved at $4^{\circ} \mathrm{C}$ in cool boxes, carried to the National Laboratory for Water Analysis in Ouagadougou and stored in a refrigerator at $4^{\circ} \mathrm{C}$ before analysis. Some samples were blocked by the addition of mercuric chloride and analyzed in Belgium for nitrates at the laboratory of water resources, University of Liège.

During sampling, field observations, supplemented by information collected from water users and local authorities fed the reflection in order to try to identify the sources of constraints to safe drinking water access.

\subsection{Sample Analysis}

A total of 15 physicochemical parameters were determined. Parameters such as $\mathrm{pH}$ and conductivity were monitored on-site with a portable Hatch Multimeter and kit. Other parameters were determined from samples preserved at $4^{\circ} \mathrm{C}$ in cool boxes by the National Laboratory for Water Analysis of the Ministry of Environment and Sustainable Development in Ouagadougou, in the 2 - 3 days following sampling, using standard methods [25, 26].

For nitrates/nitrites determination, proportioning was carried out by molecular absorption spectrophotometry through nitrates reduction in nitrites by cadmium (spectro Hach DR2400 method 8171).

Iron in the water samples was analyzed using Atomic Absorption Spectrophotometer (AAS) after pre-concentration $[25,26]$.

Two bacterial indicators of fecal contamination namely Escherichia coli, and fecal Coliforms were enumerated 3 - 4 hours following sampling at the Laboratory of Tougan hospital, using the membrane filtration technique [25]. Bacterial cells were concentrated on a 0.2 $\mu \mathrm{m}$ Millipore Membrane Filter followed by culture on the chromogenic RAPID E. COLI 2 AGAR (BIO RAD) medium which contains 2 substrates specific to the $\beta$-DGlucuronidase (Gluc) and $\beta$-D-Galactosidase (Gal) enzymes, respectively. Incubation was performed at $44.5^{\circ} \mathrm{C}$ for $24 \mathrm{~h}$. Colonies of E. coli $\left(\mathrm{Gal}^{+} / \mathrm{Gluc}^{+}\right)$appears violet to pink while other coliforms colonies stain blue.

\subsection{Statistical Method}

Data obtained were analyzed for water source and sampling period variations using the Student's t-test and XLSTAT 7.5.2 statistical software. Mean parameters concentrations were compared according to the Newman Keuls' test.

\section{Results and Discussion}

\subsection{Water Physicochemical Characteristics}

The results of the statistical analysis on the data obtained are presented in Tables 1(a) and (b) while the mean characteristics are shown in Tables 2(a) and (b).

The Student's t-test revealed that all the characteristics of water were significantly related to the sampling site ( $p$ $<0.0001)$ and period as well $(\mathrm{p}<0.0001)$, except calcium, magnesium and total hardness (Tables 1(a) and (b)). The joined effects of site and period affected also significantly $(\mathrm{p}<0.0001)$ these characteristics except total hardness $(\mathrm{p}=0.291)$. The spatio-temporal variation in response to water source and sampling period implied that water samples were collected from sources of different physicochemical characteristics which are more influenced by the period of water collection.

Mean turbidity values recorded for some water samples were higher than the national and WHO guideline value of 5 NTU for drinking water $[11,12,20]$. According to the sampling period, high turbidity of water was recorded for the drillings of Kassoum-CEG (12 NTU in 2007), Yaba (14.6 NTU in 2007) and especially Sono (104-180 NTU in 2007-2008) (Table 2(a)). For the later, the turbidity seemed related to the oxidation of iron in contact of air as indicated by the reddish-brown color observed after a few minutes subsequent to water collection into flasks (Figure 2(b)). All samples from wells showed uniformly turbidity over the WHO threshold value. Since wells are not protected, they can receive suspended material leading to turbidity increase as confirmed our field observations. As also underlined in studies, excessive turbidity may also be associated with unpleasant tastes and odors [27]. Turbidity also correlates with iron content of water samples [28] as observed at some sampling periods for water samples of Sono and Yaba drillings (Tables 2(a) and (b), Figure 2(b)). As a consequence, the water source can be rejected by populations and that corroborated the case of Sono drilling.

The mean $\mathrm{pH}$ of the water samples ranged from slightly acid (pH 5.96) to slightly basic (pH 7.70) (Table 2(a)). Although $\mathrm{pH}$ usually has no direct impact on consumers, high $\mathrm{pH}$ can affect the palatability. No healthbased guideline value has been proposed for $\mathrm{pH}$; however, an acceptable range for drinking water $\mathrm{pH}$ is from 6.5 to 8.5 [2022]. Corrosion effects may also become significant below pH 6.5 and that corroborated the case of Sono drilling, and at a less extent Yaba drilling!

Mean water conductivity values recorded ranged from $45-2465 \mu \mathrm{S} \cdot \mathrm{cm}^{-1}$ (Table 2(b)). Although no health hazard for populations was found associated to conductivity, classification of potability based on electrical conductivity ascribes $<325 \mu \mathrm{S} \cdot \mathrm{cm}^{-1}$ for fresh and potable water [29]. From the results obtained, $60 \%$ of the water sources 
Table 1. (a) Variance of the physicochemical characteristics of water from drillings and wells with regard to sampling site and sampling period. (b) Variance of the physicochemical characteristics of water from wells and drillings with regard to sampling site and period (continued).

(a)

\begin{tabular}{|c|c|c|c|c|c|c|c|c|c|c|c|c|c|c|c|}
\hline \multirow{2}{*}{$\begin{array}{l}\text { Source of } \\
\text { variation }\end{array}$} & \multirow[t]{2}{*}{$\mathrm{df}$} & \multicolumn{2}{|c|}{$\begin{array}{c}\text { Nitrates } \\
\left(\mathrm{mg} \quad \mathrm{NO}_{3}^{-} / \mathrm{l}\right)\end{array}$} & \multicolumn{2}{|c|}{$\begin{array}{c}\text { Nitrites } \\
\left(\mathrm{mg} \quad \mathrm{NO}_{2}^{-} / \mathrm{l}\right)\end{array}$} & \multicolumn{2}{|c|}{$\mathrm{pH}$} & \multicolumn{2}{|c|}{$\begin{array}{l}\text { Potassium } \\
\left(\mathrm{mg} \mathrm{K}^{+} / \mathrm{l}\right)\end{array}$} & \multicolumn{2}{|c|}{$\begin{array}{l}\text { Sodium } \\
\left(\mathrm{mg} \mathrm{Na}^{+} / 1\right)\end{array}$} & \multicolumn{2}{|c|}{$\begin{array}{c}\text { Sulfates } \\
\left(\mathrm{mg} \mathrm{SO} \mathrm{SO}_{4}^{2-} / \mathrm{l}\right)\end{array}$} & \multicolumn{2}{|c|}{$\begin{array}{l}\text { Turbidity } \\
\text { (NTU) }\end{array}$} \\
\hline & & MS & $\mathrm{p}$ & MS & $\mathrm{p}$ & MS & $\mathrm{p}$ & MS & $\mathrm{p}$ & MS & $\mathrm{p}$ & MS & $\mathrm{p}$ & MS & $\mathrm{p}$ \\
\hline
\end{tabular}

\begin{tabular}{|c|c|c|c|c|c|c|c|}
\hline Site & 14 & $312192.8<0.0001^{* *}$ & $4882.4<0.0001^{* *} 0.982<0.0001^{* *} 1$ & $13572.61<0.0001^{* *}$ & $195.65<0.0001^{* *}$ & $868184.6<0.0001^{* *}$ & * $5202.21<0.0001^{* *}$ \\
\hline Period & 2 & $5512.7<0.0001^{* *}$ & $0.002<0.0001^{* *} \quad 0.128<0.004^{* *}$ & $540.16<0.0001^{* *}$ & $41.27<0.0001^{* *}$ & $1371.13<0.0001^{* *}$ & $24.24<0.0001^{* *}$ \\
\hline Period & 28 & $4882.4<0.0001^{* *}$ & $0.001<0.0001^{* *} 0.101<0.0001^{* *}$ & $371.42<0.0001^{* *}$ & $3.25<0.0001^{* *}$ & $1052.77<0.0001^{* *}$ & $1600.09<0.0001^{* *}$ \\
\hline
\end{tabular}

MS means square; ${ }^{* *}$ significant $\mathrm{p}<0.01$.

(b)

\begin{tabular}{|c|c|c|c|c|c|c|c|c|c|c|c|c|c|c|c|c|c|}
\hline \multirow{2}{*}{$\begin{array}{l}\text { Source of } \\
\text { variation }\end{array}$} & \multirow[t]{2}{*}{$\mathrm{df}$} & \multicolumn{2}{|c|}{$\begin{array}{l}\text { Ammonium } \\
\left(\mathrm{mg} \mathrm{NH}_{4}^{+} / 1\right)\end{array}$} & \multicolumn{2}{|c|}{$\begin{array}{l}\text { Conductivity } \\
(\mu \mathrm{S} / \mathrm{cm})\end{array}$} & \multicolumn{2}{|c|}{$\begin{array}{l}\text { Arsenic } \\
(\mu \mathrm{g} \mathrm{As} / \mathrm{L})\end{array}$} & \multicolumn{2}{|c|}{$\begin{array}{c}\text { Calcium } \\
\left(\mathrm{mg} \mathrm{Ca}^{2+} / 1\right)\end{array}$} & \multicolumn{2}{|c|}{$\begin{array}{c}\text { Calcium } \\
\text { hardness } \\
\left(\mathrm{mg} \mathrm{CaCO}_{3} / \mathrm{l}\right)\end{array}$} & \multicolumn{2}{|c|}{$\begin{array}{l}\text { Total hardness } \\
\left(\mathrm{mg} \mathrm{CaCO}_{3} / \mathrm{l}\right)\end{array}$} & \multicolumn{2}{|c|}{$\begin{array}{l}\text { Total Iron } \\
(\mathrm{mg} \mathrm{Fe} / \mathrm{l})\end{array}$} & \multicolumn{2}{|c|}{$\begin{array}{l}\text { Magnesium } \\
\left(\mathrm{mg} \mathrm{Mg}^{2+} / 1\right)\end{array}$} \\
\hline & & MS & $\mathrm{p}$ & MS & $\mathrm{p}$ & MS & $\mathrm{p}$ & MS & $\mathrm{p}$ & MS & $\mathrm{p}$ & MS & $\mathrm{p}$ & MS & $\mathrm{p}$ & MS & $\mathrm{p}$ \\
\hline Site & 14 & $1.993<$ & $0001^{* *}$ & \multicolumn{2}{|c|}{$\begin{array}{c}3572554.2< \\
0.0001^{* *}\end{array}$} & \multicolumn{2}{|c|}{$\begin{array}{l}1.548< \\
0.0001^{* *}\end{array}$} & \multicolumn{2}{|c|}{$\begin{array}{c}89167.4< \\
0.0001^{* *}\end{array}$} & \multicolumn{2}{|c|}{$\begin{array}{c}553279< \\
0.0001^{* *}\end{array}$} & \multicolumn{2}{|c|}{$\begin{array}{c}1136052.2< \\
0.0001^{* *}\end{array}$} & \multicolumn{2}{|c|}{$\begin{array}{l}27.17< \\
0.0001^{* *}\end{array}$} & \multicolumn{2}{|c|}{$\begin{array}{c}7238.99< \\
0.0001^{* *}\end{array}$} \\
\hline Period & 2 & 0.035 & $0001^{* *}$ & 6154.45 & $.0001^{* *}$ & \multicolumn{2}{|c|}{$\begin{array}{l}2.118< \\
0.0001^{* *}\end{array}$} & 2.164 & $0.754^{\mathrm{ns}}$ & 74.63 & $0.018^{*}$ & \multicolumn{2}{|c|}{$7329.800 .0383^{\mathrm{ns}}$} & \multicolumn{2}{|c|}{$61.150 .0001^{* *}$} & \multicolumn{2}{|c|}{$14.920 .038^{*}$} \\
\hline Site*Period & 28 & $0.020<$ & $0001^{* *}$ & 16955.9 & $.0001^{* *}$ & \multicolumn{2}{|c|}{$\begin{array}{l}0.239< \\
0.0001^{* *}\end{array}$} & \multicolumn{2}{|c|}{$\begin{array}{c}40.929< \\
0.0001^{* *}\end{array}$} & \multicolumn{2}{|c|}{$\begin{array}{l}233.61< \\
0.0001^{* *}\end{array}$} & 8792.11 & $0.291^{\mathrm{ns}}$ & \multicolumn{2}{|c|}{$\begin{array}{l}15.38< \\
0.0001^{* *}\end{array}$} & \multicolumn{2}{|c|}{$31.45<0.0001^{* *}$} \\
\hline
\end{tabular}

MS means square; ${ }^{*}$ significant $\mathrm{p}<0.05 ;{ }^{* *}$ significant $\mathrm{p}<0.01 ;{ }^{\mathrm{ns}}$ not significant $\mathrm{p}<0.05$.

showed mean values of water electrical conductivity over the ascribed potability value of $325 \mu \mathrm{S} \cdot \mathrm{cm}^{-1}$. Mean values were particularly high throughout the 3 sampling periods for the drillings of Wawara $\left(2215-2465 \mu \mathrm{S} \cdot \mathrm{cm}^{-1}\right)$, Diouroum (837 - $\left.1168 \mu \mathrm{S} \cdot \mathrm{cm}^{-1}\right)$; Di $\left(764-906 \mu \mathrm{S} \cdot \mathrm{cm}^{-1}\right)$, Niassan-AMVS $\left(541-606 \mu \mathrm{S} \cdot \mathrm{cm}^{-1}\right)$ and Niassan-Clinic $\left(557-600 \mu \mathrm{S} \cdot \mathrm{cm}^{-1}\right)$. The high conductivity of these ground-waters may be related to the bedrock they flow through as suggested [21].

Total Hardness values in the water samples ranged from 16.0 - $1447.3 \mathrm{mg} / \mathrm{CaCO}_{3}$. This parameter varied significantly with only the sampling source $(\mathrm{p}<0.0001)$ but not with the sampling period $(\mathrm{p}=0.383$ ) or both joined factors $(p=0.291)$. Water hardness was particularly remarkable for all the water sources studied, except the drilling of Kouy, the modern well of Yaba and the traditional well of Sono. A high and practically constant value throughout the study periods was recorded for the water of Wawara drilling (1446 - $\left.1447 \mathrm{mg} \mathrm{CaCO}_{3} / \mathrm{l}\right)$. Hardness in water comprises the determination of calcium and magnesium as the main constituents and their widespread abundance in rock formations leads often to very considerable hardness levels in surface waters or groundwaters. One of several arbitrary classifications of waters by hardness includes: Soft, up to $50 \mathrm{mg} / \mathrm{l}$; Moder- ately Soft, 51 - 100 mg/l; Slightly Hard, 101 - 150 /1; Moderately Hard, 151 - 250 mg/l; Hard, 251 - 350 mg/l; Excessively Hard, over $350 \mathrm{mg} / 1$ [30]. The degree of hardness of drinking water is important for aesthetic acceptability by consumers.

From the values recorded (Table 2(b)), waters were classified as Soft for the drilling of Kouy (18 - $20 \mathrm{mg}$ $\mathrm{CaCO}_{3} / 1$ ), the modern well of Kassoum-Market (25 $\left.35.3 \mathrm{mg} \mathrm{CaCO}_{3} / \mathrm{l}\right)$, and the traditional well of KouyMosque (16 - $\left.22.6 \mathrm{mg} \mathrm{CaCO}_{3} / \mathrm{l}\right)$; Moderately Soft for the drilling of Sono-Clinic (90.3 - $\left.91 \mathrm{mg} \mathrm{CaCO}_{3} / \mathrm{l}\right)$ and the traditional well of Sono-Centre $\left(62-64 \mathrm{mg} \mathrm{CaCO}_{3} / \mathrm{l}\right)$, Slightly Hard for the drillings of Kassoum-CEG (107.6 $\left.128.6 \mathrm{mg} \mathrm{CaCO}_{3} / \mathrm{l}\right)$ and Yaba-Clinic (142 - $146 \mathrm{mg}$ $\left.\mathrm{CaCO}_{3} / 1\right)$; Slightly-to-Moderately Hard for the drilling of Kassoum-School (117 - $\left.240 \mathrm{mg} \mathrm{CaCO}_{3} / \mathrm{l}\right)$, Moderately Hard for the modern well of Yaba-Clinic (155 - 157.3 $\left.\mathrm{mg} \mathrm{CaCO}_{3} / 1\right)$, Hard for the drillings of Di (323.6 - 326 mg $\left.\mathrm{CaCO}_{3} / \mathrm{l}\right)$, Niassan-AMVS (271 - $\left.318.6 \mathrm{mg} \mathrm{CaCO}_{3} / \mathrm{l}\right)$ and Niassan-Clinic (310 - $\left.320 \mathrm{mg} \mathrm{CaCO}_{3} / \mathrm{l}\right)$; Slightlyto-Excessively Hard for the modern well of Diouroum (113.6 - $\left.453 \mathrm{mg} \mathrm{CaCO}_{3} / \mathrm{l}\right)$ and Excessively Hard for the drillings of Diouroum (490 - $\left.535.3 \mathrm{mg} \mathrm{CaCO}_{3} / \mathrm{l}\right)$ and Wawara (1446.3 - $\left.1447.3 \mathrm{mg} \mathrm{CaCO}_{3} / \mathrm{l}\right)$. Although there is evidence from epidemiological studies for a protective 
Table 2. (a) Mean ${ }^{*}$ physicochemical characteristics of water from wells and drillings during the dry season in 2007, 2008 and 2012 ("mean of 3 replicates); (b) Mean* physicochemical characteristics of water from wells and drillings during the dry season in 2007, 2008 and 2012 ('mean of 3 replicates).

(a)

\begin{tabular}{|c|c|c|c|c|c|c|c|c|}
\hline Sampling Site & Period & $\begin{array}{c}\text { Nitrates } \\
\left(\mathrm{mg} \mathrm{NO}_{3}^{-} / 1\right)\end{array}$ & $\begin{array}{c}\text { Nitrites } \\
\left(\mathrm{mg} \mathrm{NO} \mathrm{NO}_{2}^{-} / 1\right)\end{array}$ & $\mathrm{pH}$ & $\begin{array}{l}\text { Potassium } \\
\left(\mathrm{mg} \mathrm{K}^{+} / 1\right)\end{array}$ & $\begin{array}{c}\text { Sodium } \\
\left(\mathrm{mg} \mathrm{Na}^{+} / 1\right)\end{array}$ & $\begin{array}{c}\text { Sulfates } \\
\left(\mathrm{mg} \mathrm{SO} \mathrm{SO}_{4}^{2-} / 1\right)\end{array}$ & Turbidity (NTU) \\
\hline \multirow{3}{*}{$\mathrm{Di}-\mathrm{CP}^{\mathrm{D}}$} & 2007 & $115^{\mathrm{g}}$ & $0.027^{\text {defghi }}$ & $6.9^{\text {bcdefgh }}$ & $55^{\mathrm{f}}$ & $7^{\mathrm{g}}$ & $38^{\mathrm{c}}$ & $1^{\mathrm{h}}$ \\
\hline & 2008 & $126.5^{\mathrm{ef}}$ & $0.013^{\text {ghi }}$ & $6.8^{\text {cdefghi }}$ & $120^{\mathrm{c}}$ & $2.2^{\mathrm{klmn}}$ & $40^{\mathrm{c}}$ & $1^{\mathrm{h}}$ \\
\hline & 2012 & $128^{\mathrm{ef}}$ & $0.017^{\mathrm{fghi}}$ & $7.0^{\text {bcde }}$ & $90^{\mathrm{d}}$ & $5^{\text {hi }}$ & $42^{c}$ & $1.16^{\text {gh }}$ \\
\hline \multirow{3}{*}{ Dioroum-COPROD ${ }^{\mathrm{D}}$} & 2007 & $860^{\mathrm{a}}$ & $0.200^{\mathrm{b}}$ & $6.46^{\mathrm{hijklm}}$ & $6^{\mathrm{klm}}$ & $14^{\mathrm{c}}$ & $43^{\mathrm{c}}$ & $1^{\mathrm{h}}$ \\
\hline & 2008 & $610^{\mathrm{c}}$ & $0.187^{\mathrm{b}}$ & $6.46^{\mathrm{hijklm}}$ & $5.03^{\mathrm{klm}}$ & $9.1^{\mathrm{e}}$ & $45^{\mathrm{c}}$ & $1^{\mathrm{h}}$ \\
\hline & 2012 & $751.7^{\mathrm{b}}$ & $0.290^{\mathrm{a}}$ & $6.10^{\text {no }}$ & $5.13^{\mathrm{klm}}$ & $12^{\mathrm{d}}$ & $42^{\mathrm{c}}$ & $1^{\mathrm{h}}$ \\
\hline \multirow{3}{*}{ Kassoum-CEG ${ }^{\mathrm{D}}$} & 2007 & $3^{\mathrm{q}}$ & $0.040^{\text {cdef }}$ & $6.6^{\text {efghijkl }}$ & $3^{\operatorname{lm}}$ & $0.86^{\mathrm{no}}$ & $5.66^{\mathrm{ij}}$ & $12^{\mathrm{ef}}$ \\
\hline & 2008 & $2.23^{\mathrm{q}}$ & $0.014^{\mathrm{ghi}}$ & $6.6^{\text {efghijkl }}$ & $4.2^{\mathrm{klm}}$ & $0.53^{\circ}$ & $7^{\text {hij }}$ & $2.63^{\mathrm{gh}}$ \\
\hline & 2012 & $3^{\mathrm{q}}$ & $0.011^{\text {ghi }}$ & $6.43^{\mathrm{ijklmn}}$ & $2.8^{\mathrm{lm}}$ & $0.83^{\mathrm{no}}$ & $6^{\mathrm{ij}}$ & $3^{\text {gh }}$ \\
\hline \multirow{3}{*}{ Kassoum-School $^{\mathrm{D}}$} & 2007 & $19.3^{\operatorname{lmn}}$ & $0.040^{\text {cdef }}$ & $7.13^{\mathrm{bc}}$ & $6^{\mathrm{klm}}$ & $2^{\mathrm{klmno}}$ & $1.6^{\mathrm{j}}$ & $1^{\mathrm{h}}$ \\
\hline & 2008 & $23^{\operatorname{lm}}$ & $0.035^{\operatorname{defg}}$ & $7.0^{\text {bcde }}$ & $2.6^{\mathrm{Im}}$ & $1.5^{\mathrm{klmno}}$ & $2.3^{\mathrm{j}}$ & $1.3^{\mathrm{gh}}$ \\
\hline & 2012 & $24^{1}$ & $0.026^{\text {efghi }}$ & $6.96^{\text {bcdef }}$ & $5.7^{\mathrm{klm}}$ & $1.8^{\mathrm{klmno}}$ & $2^{\mathrm{j}}$ & $1^{\mathrm{h}}$ \\
\hline \multirow{3}{*}{ Kouy-COPROD ${ }^{\mathrm{D}}$} & 2007 & $19.5^{\operatorname{lmn}}$ & $0.004^{\mathrm{hi}}$ & $6.43^{\mathrm{ijklmn}}$ & $0.20^{\mathrm{m}}$ & $0.73^{\text {no }}$ & $1.7^{\mathrm{j}}$ & $2.76^{\mathrm{gh}}$ \\
\hline & 2008 & $19.9^{\operatorname{lmn}}$ & $0.004^{\mathrm{hi}}$ & $6.50^{\text {ghijklmn }}$ & $0.25^{\mathrm{m}}$ & $0.8^{\mathrm{no}}$ & $1.5^{\mathrm{j}}$ & $2.73^{\mathrm{gh}}$ \\
\hline & 2012 & $13.8^{\text {Imnopq }}$ & $0.002^{\mathrm{i}}$ & $6.63^{\text {efghijk }}$ & $0.23^{\mathrm{m}}$ & $0.86^{\mathrm{no}}$ & $1.76^{\mathrm{j}}$ & $2.3^{\mathrm{gh}}$ \\
\hline \multirow{3}{*}{ Niassan-AMVS ${ }^{\mathrm{D}}$} & 2007 & $12.1^{1 \mathrm{mnopq}}$ & $0.020^{\mathrm{fghi}}$ & $7.0^{\text {bcde }}$ & $4^{\mathrm{klm}}$ & $8.3^{\text {ef }}$ & $13.5^{\mathrm{fghi}}$ & $1^{\mathrm{h}}$ \\
\hline & 2008 & $12.6^{\operatorname{lmnopq}}$ & $0.016^{\mathrm{fghi}}$ & $7.0^{\text {bcde }}$ & $2.5^{\operatorname{lm}}$ & $4.7^{\mathrm{hi}}$ & $14.16^{\text {fghi }}$ & $1.3^{\mathrm{gh}}$ \\
\hline & 2012 & $18.3^{\operatorname{lmn}}$ & $0.030^{\text {defgh }}$ & $7.0^{\text {bcde }}$ & $3.1^{\mathrm{lm}}$ & $9^{\mathrm{e}}$ & $15^{\text {efghi }}$ & $1.2^{\mathrm{gh}}$ \\
\hline \multirow{3}{*}{ Niassan-Clinic ${ }^{\mathrm{D}}$} & 2007 & $11^{\mathrm{mnopq}}$ & $0.010^{\text {ghi }}$ & $7.1^{\mathrm{bcd}}$ & $3^{\operatorname{lm}}$ & $9^{\mathrm{e}}$ & $13^{\text {fghi }}$ & $1^{\mathrm{h}}$ \\
\hline & 2008 & $15.3^{\operatorname{lmnop}}$ & $0.010^{\text {ghi }}$ & $7.7^{\mathrm{a}}$ & $4.4^{\mathrm{klm}}$ & $4.9^{\mathrm{hi}}$ & $14^{\text {fghi }}$ & $1.5^{\mathrm{gh}}$ \\
\hline & 2012 & $12.1^{1 \mathrm{mnopq}}$ & $0.010^{\text {ghi }}$ & $7.0^{\text {bcde }}$ & $5^{\mathrm{klm}}$ & $7^{\mathrm{g}}$ & $16^{\text {defgh }}$ & $4^{\text {gh }}$ \\
\hline \multirow{3}{*}{ Sono-Clinic ${ }^{\mathrm{D}}$} & 2007 & $3^{\mathrm{q}}$ & $0.040^{\text {cdef }}$ & $6.26^{\mathrm{klmno}}$ & $2^{\mathrm{m}}$ & $3^{\mathrm{jk}}$ & $6^{\mathrm{ij}}$ & $180^{\mathrm{a}}$ \\
\hline & 2008 & $4^{\mathrm{pq}}$ & $0.023^{\text {efghi }}$ & $6.16^{\mathrm{mno}}$ & $1.9^{\mathrm{m}}$ & $1.73^{\mathrm{klmno}}$ & $5.33^{\mathrm{ij}}$ & $104^{\mathrm{b}}$ \\
\hline & 2012 & $4.23^{\mathrm{pq}}$ & $0.012^{\text {ghi }}$ & $6.66^{\text {defghijk }}$ & $2.5^{\operatorname{lm}}$ & $2.76^{\mathrm{jkl}}$ & $8^{\text {ghij }}$ & $2.26^{\mathrm{gh}}$ \\
\hline \multirow{3}{*}{ Yaba-Clinic $^{\mathrm{D}}$} & 2007 & $122.3^{\mathrm{f}}$ & $0.013^{\text {ghi }}$ & $6.03^{\circ}$ & $15^{\mathrm{j}}$ & $9^{\mathrm{e}}$ & $24^{\mathrm{de}}$ & $14.66^{\mathrm{de}}$ \\
\hline & 2008 & $134^{\mathrm{e}}$ & $0.018^{\text {fghi }}$ & $6.20^{\operatorname{lmno}}$ & $5.2^{\mathrm{klm}}$ & $5.2^{\mathrm{hi}}$ & $22^{\text {def }}$ & $2.8^{\mathrm{gh}}$ \\
\hline & 2012 & $82.7^{\mathrm{i}}$ & $0.027^{\text {fghi }}$ & $5.96^{\circ}$ & $6^{\mathrm{klm}}$ & $7.5^{\mathrm{fg}}$ & $25^{\mathrm{d}}$ & $4^{\text {gh }}$ \\
\hline \multirow{3}{*}{ Wawara-COPROD ${ }^{\mathrm{D}}$} & 2007 & $5.23^{\mathrm{opq}}$ & $0.010^{\text {ghi }}$ & $6.73^{\text {cdefghij }}$ & $130^{\mathrm{ab}}$ & $17^{\mathrm{b}}$ & $1175^{\mathrm{b}}$ & $1.2^{\mathrm{gh}}$ \\
\hline & 2008 & $5.20^{\mathrm{opq}}$ & $0.013^{\text {ghi }}$ & $6.70^{\text {cdefghijk }}$ & $132^{\mathrm{a}}$ & $17^{\mathrm{b}}$ & $1175^{\mathrm{b}}$ & $1.2^{\mathrm{gh}}$ \\
\hline & 2012 & $2.5^{\mathrm{q}}$ & $0.042^{\text {cdef }}$ & $6.66^{\text {defghijk }}$ & $128^{\mathrm{b}}$ & $19^{\mathrm{a}}$ & $1301.66^{\mathrm{a}}$ & $4^{\text {gh }}$ \\
\hline \multirow{3}{*}{ Dioroum-PNGT ${ }^{\mathrm{MW}}$} & 2007 & $37.3^{\mathrm{k}}$ & $0.021^{\text {fghi }}$ & $7.20^{\mathrm{b}}$ & $32.3^{\mathrm{i}}$ & $2^{\mathrm{klmno}}$ & $16^{\text {defgh }}$ & $5^{\text {gh }}$ \\
\hline & 2008 & $96.7^{\mathrm{h}}$ & $0.022^{\text {fghi }}$ & $7.20^{\mathrm{b}}$ & $80^{\mathrm{e}}$ & $1^{\mathrm{mno}}$ & $17^{\text {defg }}$ & $2.5^{\mathrm{gh}}$ \\
\hline & 2012 & $45.2^{\mathrm{jk}}$ & $0.025^{\mathrm{eghhi}}$ & $6.93^{\text {bcdefg }}$ & $30^{\mathrm{i}}$ & $2^{\mathrm{klmno}}$ & $18^{\mathrm{def}}$ & $5.16^{\mathrm{gh}}$ \\
\hline
\end{tabular}


Continued

\begin{tabular}{|c|c|c|c|c|c|c|c|c|}
\hline \multirow{3}{*}{ Kassoum-Market $^{\mathrm{MW}}$} & 2007 & $15^{\mathrm{Imnop}}$ & $0.010^{\mathrm{ghi}}$ & $6.7^{\text {cdefghijk }}$ & $1^{\mathrm{m}}$ & $3^{\mathrm{jk}}$ & $2^{j}$ & $4^{\text {gh }}$ \\
\hline & 2008 & $13.7^{\mathrm{Imnopq}}$ & $0.010^{\text {ghi }}$ & $6.8^{\text {cdefghi }}$ & $0.9^{\mathrm{m}}$ & $1.3^{\operatorname{lmno}}$ & $2^{j}$ & $2.4^{\mathrm{gh}}$ \\
\hline & 2012 & $10.4^{\text {nopq }}$ & $0.010^{\mathrm{ghi}}$ & $6.8^{\text {cdefghi }}$ & $3^{\operatorname{lm}}$ & $1.5^{\mathrm{klmno}}$ & $2^{j}$ & $6.43^{\mathrm{g}}$ \\
\hline \multirow{3}{*}{ Yaba-Clinic $^{\mathrm{PM}}$} & 2007 & $240^{\mathrm{d}}$ & $0.050^{\mathrm{cd}}$ & $6.30^{\mathrm{jklmno}}$ & $50^{\mathrm{g}}$ & $5.1^{\mathrm{hi}}$ & $39^{\mathrm{c}}$ & $2.5^{\mathrm{gh}}$ \\
\hline & 2008 & $93^{\mathrm{h}}$ & $0.020^{\text {fghi }}$ & $6.30^{\mathrm{jklmno}}$ & $50.7^{\mathrm{g}}$ & $5.1^{\mathrm{hi}}$ & $40^{\mathrm{c}}$ & $3^{\text {gh }}$ \\
\hline & 2012 & $97.3^{\mathrm{h}}$ & $0.060^{\mathrm{c}}$ & $6.63^{\text {efghijk }}$ & $44.7^{\mathrm{h}}$ & $5.8^{\mathrm{h}}$ & $43^{c}$ & $4.9^{\mathrm{gh}}$ \\
\hline \multirow{3}{*}{ Kouy-Mosque ${ }^{\mathrm{TW}}$} & 2007 & $16^{\mathrm{lmno}}$ & $0.030^{\mathrm{defgh}}$ & $6.7^{\text {cdefghijk }}$ & $1^{\mathrm{m}}$ & $4^{\mathrm{ij}}$ & $1.63^{\mathrm{j}}$ & $23.66^{\mathrm{c}}$ \\
\hline & 2008 & $18.5^{\mathrm{lmn}}$ & $0.025^{\text {efghi }}$ & $6.9^{\text {bcdefgh }}$ & $0.6^{\mathrm{m}}$ & $2.8^{\mathrm{jkl}}$ & $1.8^{\mathrm{j}}$ & $22^{\mathrm{c}}$ \\
\hline & 2012 & $20.5^{\operatorname{lmn}}$ & $0.047^{\text {cde }}$ & $6.20^{\operatorname{lmno}}$ & $0.8^{\mathrm{m}}$ & $3^{\mathrm{jk}}$ & $2^{j}$ & $23.36^{\mathrm{c}}$ \\
\hline \multirow{3}{*}{ Sono-Centre ${ }^{\mathrm{TW}}$} & 2007 & $46^{\mathrm{jk}}$ & $0.010^{\text {ghi }}$ & $6.70^{\text {cdefghijk }}$ & $9^{k}$ & $2^{\mathrm{klmno}}$ & $6^{\mathrm{ij}}$ & $15^{\mathrm{de}}$ \\
\hline & 2008 & $50.5^{\mathrm{j}}$ & $0.018^{\mathrm{fghi}}$ & $6.80^{\text {cdefghi }}$ & $4.3^{\mathrm{klm}}$ & $1.66^{\mathrm{klmno}}$ & $5^{\mathrm{ij}}$ & $11.16^{\mathrm{f}}$ \\
\hline & 2012 & $37.3^{\mathrm{k}}$ & $0.010^{\text {ghi }}$ & $6.53^{\text {fghijklm }}$ & $8^{\mathrm{kl}}$ & $2.53^{\mathrm{klm}}$ & $8^{\text {ghij }}$ & $16.86^{\mathrm{d}}$ \\
\hline \multicolumn{2}{|c|}{ Guideline values [20-22] } & 50 & 3 & $6.5-8.5$ & 12 & $\leq 200$ & $\leq 500$ & $\leq 5$ \\
\hline
\end{tabular}

Means with a same letter within a column are not significantly different according to Newman Keuls' test $\mathrm{p}<0.05 .{ }^{\mathrm{D}}$ drilling; ${ }^{\mathrm{MW}}$ modern well; ${ }^{\mathrm{TW}}$ traditional well.

(b)

\begin{tabular}{|c|c|c|c|c|c|c|c|c|c|}
\hline Sampling Site & Period & $\begin{array}{l}\text { Ammonium } \\
\left(\mathrm{mg} \mathrm{NH}_{4}^{+} / \mathrm{l}\right)\end{array}$ & $\begin{array}{l}\text { Conductivity } \\
(\mu \mathrm{S} / \mathrm{cm})\end{array}$ & $\begin{array}{l}\text { Arsenic } \\
(\mu \mathrm{g} \mathrm{As} / 1)\end{array}$ & $\begin{array}{l}\text { Calcium } \\
\left(\mathrm{mg} \mathrm{Ca}^{2+} / \mathrm{l}\right)\end{array}$ & $\begin{array}{l}\text { Calcium hardness } \\
\left(\mathrm{mg} \mathrm{CaCO}_{3} / \mathrm{l}\right)\end{array}$ & $\begin{array}{l}\text { Total hardness } \\
\left(\mathrm{mg} \mathrm{CaCO}_{3} / \mathrm{l}\right)\end{array}$ & $\begin{array}{l}\text { Total Iron } \\
(\mathrm{mg} \mathrm{Fe} / \mathrm{l})\end{array}$ & $\begin{array}{l}\text { Magnesium } \\
\left(\mathrm{mg} \mathrm{Mg}^{2+} / \mathrm{l}\right)\end{array}$ \\
\hline \multirow{3}{*}{ Di-CP ${ }^{\mathrm{D}}$} & 2007 & $0.030^{\text {nop }}$ & $865^{\mathrm{f}}$ & $2^{\mathrm{bc}}$ & $68^{\mathrm{d}}$ & $171^{\text {def }}$ & $323.6^{\text {bcde }}$ & $0.09^{\mathrm{jk}}$ & $37^{\mathrm{e}}$ \\
\hline & 2008 & $0.030^{\text {nop }}$ & $906^{\mathrm{e}}$ & $1^{\mathrm{d}}$ & $70^{\mathrm{d}}$ & $174^{\mathrm{de}}$ & $325^{\text {bcde }}$ & $0.1^{\mathrm{jk}}$ & $38^{\mathrm{e}}$ \\
\hline & 2012 & $0.030^{\text {nop }}$ & $764^{\mathrm{h}}$ & $2^{\mathrm{bc}}$ & $66^{\mathrm{d}}$ & $176^{\mathrm{d}}$ & $326^{\text {bcde }}$ & $0.09^{\mathrm{jk}}$ & $36^{\mathrm{e}}$ \\
\hline \multirow{3}{*}{ Dioroum-COPROD ${ }^{\mathrm{D}}$} & 2007 & $0.430^{\mathrm{e}}$ & $1079.3^{\mathrm{d}}$ & $2^{\mathrm{bc}}$ & $92^{\mathrm{c}}$ & $231^{\mathrm{c}}$ & $490^{\mathrm{bc}}$ & $0.03^{\mathrm{k}}$ & $62^{\mathrm{d}}$ \\
\hline & 2008 & $0.553^{d}$ & $1168.6^{\mathrm{c}}$ & $1.33^{\mathrm{cd}}$ & $100^{\mathrm{b}}$ & $249^{b}$ & $535.3^{\mathrm{b}}$ & $9.8^{\mathrm{a}}$ & $70^{\mathrm{c}}$ \\
\hline & 2012 & $0.380^{\mathrm{f}}$ & $837.6^{\mathrm{g}}$ & $1.66^{\mathrm{bcd}}$ & $95^{\mathrm{c}}$ & $235^{\mathrm{c}}$ & $501.6^{\mathrm{bc}}$ & $0.02^{\mathrm{k}}$ & $65^{\mathrm{d}}$ \\
\hline \multirow{3}{*}{ Kassoum-CEG ${ }^{\mathrm{D}}$} & 2007 & $0.050^{\mathrm{mnop}}$ & $207.6^{\mathrm{vwu}}$ & $2^{\mathrm{bc}}$ & $20^{\mathrm{kl}}$ & $50^{\mathrm{mn}}$ & $107.6^{\mathrm{ef}}$ & $0.05^{\mathrm{jk}}$ & $14^{\mathrm{hijkl}}$ \\
\hline & 2008 & $0.130^{\mathrm{ijklm}}$ & $257.6^{\mathrm{u}}$ & $1^{\mathrm{d}}$ & $26^{\text {hij }}$ & $63.6^{1}$ & $128.6^{\mathrm{ef}}$ & $0.2^{\mathrm{ijk}}$ & $16^{\mathrm{hij}}$ \\
\hline & 2012 & $0.080^{\mathrm{jklmnop}}$ & $260^{\mathrm{u}}$ & $1^{\mathrm{d}}$ & $23.33^{\mathrm{ijk}}$ & $55^{\mathrm{m}}$ & $111.6^{\mathrm{ef}}$ & $0.02^{\mathrm{k}}$ & $15^{\mathrm{hijk}}$ \\
\hline \multirow{3}{*}{ Kassoum-School $^{\mathrm{D}}$} & 2007 & $0.080^{\mathrm{jklmnop}}$ & $440.3^{\mathrm{n}}$ & $1.66^{\mathrm{bcd}}$ & $44^{\mathrm{e}}$ & $110.3^{\mathrm{i}}$ & $240^{\operatorname{def}}$ & $0.17^{\mathrm{jk}}$ & $31.3^{\mathrm{f}}$ \\
\hline & 2008 & $0.070^{\mathrm{klmnop}}$ & $206.6^{\mathrm{vwu}}$ & $1^{\mathrm{d}}$ & $22^{\mathrm{kl}}$ & $55^{\mathrm{m}}$ & $117^{\mathrm{ef}}$ & $0.3^{\mathrm{ijk}}$ & $15^{\mathrm{hijk}}$ \\
\hline & 2012 & $0.063^{\mathrm{Imnop}}$ & $332.3^{t}$ & $1^{\mathrm{d}}$ & $35^{\mathrm{fg}}$ & $80^{\mathrm{jk}}$ & $202^{\text {ef }}$ & $2.5^{\mathrm{g}}$ & $18^{\mathrm{h}}$ \\
\hline \multirow{3}{*}{ Kouy-COPROD ${ }^{\mathrm{D}}$} & 2007 & $0.020^{\mathrm{op}}$ & $45^{z}$ & $1^{\mathrm{d}}$ & $4^{\circ}$ & $10.3^{\mathrm{qr}}$ & $18.6^{\mathrm{f}}$ & $1.3^{\mathrm{h}}$ & $2.5^{\mathrm{m}}$ \\
\hline & 2008 & $0.003^{p}$ & $46^{\mathrm{z}}$ & $1^{\mathrm{d}}$ & $4^{\circ}$ & $11.6^{\mathrm{qr}}$ & $18^{\mathrm{f}}$ & $1.5^{\mathrm{h}}$ & $2.23^{\mathrm{m}}$ \\
\hline & 2012 & $0.002^{\mathrm{p}}$ & $54.6^{\mathrm{z}}$ & $1^{\mathrm{d}}$ & $5^{\circ}$ & $11.3^{\mathrm{qr}}$ & $20^{\mathrm{f}}$ & $0.29^{\mathrm{ijk}}$ & $2.26^{\mathrm{m}}$ \\
\hline \multirow{3}{*}{ Niassan-AMVS ${ }^{\mathrm{D}}$} & 2007 & $0.050^{\mathrm{mnop}}$ & $580^{\mathrm{j}}$ & $1.93^{\mathrm{bc}}$ & $63^{\mathrm{d}}$ & $157.6^{\mathrm{g}}$ & $282.6^{\text {cdef }}$ & $0.02^{\mathrm{k}}$ & $39^{\mathrm{e}}$ \\
\hline & 2008 & $0.160^{\mathrm{ij}}$ & $606.3^{\mathrm{i}}$ & $1^{\mathrm{d}}$ & $69^{\mathrm{d}}$ & $170.6^{\text {def }}$ & $318.6^{\text {bcde }}$ & $0.35^{\mathrm{ijk}}$ & $26^{\mathrm{g}}$ \\
\hline & 2012 & $0.143^{\mathrm{ijkl}}$ & $541.3^{1}$ & $1^{\mathrm{d}}$ & $67^{\mathrm{d}}$ & $165^{\text {efg }}$ & $271^{\text {cdef }}$ & $0.03^{\mathrm{k}}$ & $30^{\mathrm{f}}$ \\
\hline
\end{tabular}


Continued

\begin{tabular}{|c|c|c|c|c|c|c|c|c|c|}
\hline & 2007 & $0.053^{\mathrm{mnop}}$ & $582.3^{\mathrm{j}}$ & $1.93^{\mathrm{bc}}$ & $66^{\mathrm{d}}$ & $163^{\mathrm{fg}}$ & $315^{\text {bcde }}$ & $0.02^{\mathrm{k}}$ & $37^{\mathrm{e}}$ \\
\hline \multirow[t]{3}{*}{ Niassan-Clinic ${ }^{\mathrm{D}}$} & 2008 & $0.060^{\operatorname{lmnop}}$ & $600^{\mathrm{i}}$ & $1.06^{\mathrm{d}}$ & $66.6^{\mathrm{d}}$ & $170^{\mathrm{def}}$ & $320^{\text {bcde }}$ & $7^{\mathrm{c}}$ & $37^{\mathrm{e}}$ \\
\hline & 2012 & $0.080^{\mathrm{jklmnop}}$ & $557.3^{\mathrm{k}}$ & $2^{\mathrm{bc}}$ & $65^{\mathrm{d}}$ & $150^{\mathrm{h}}$ & $310^{\text {bcde }}$ & $0.02^{\mathrm{k}}$ & $35^{\mathrm{e}}$ \\
\hline & 2007 & $0.553^{\mathrm{d}}$ & $217.3^{\mathrm{v}}$ & $1.66^{\mathrm{bcd}}$ & $17^{\mathrm{klm}}$ & $42.6^{\text {no }}$ & $91^{\mathrm{ef}}$ & $3.5^{\mathrm{e}}$ & $12^{\mathrm{ijkl}}$ \\
\hline \multirow[t]{3}{*}{ Sono-Clinic ${ }^{\mathrm{D}}$} & 2008 & $0.703^{c}$ & $204.6^{\mathrm{vwu}}$ & $1^{\mathrm{d}}$ & $15^{\mathrm{lm}}$ & $38^{\circ}$ & $90.3^{\text {ef }}$ & $2.8^{\mathrm{f}}$ & $13^{\mathrm{hijk}}$ \\
\hline & 2012 & $0.700^{c}$ & $147.3^{y}$ & $1^{\mathrm{d}}$ & $16^{\operatorname{lm}}$ & $40.3^{\text {no }}$ & $90.3^{\text {ef }}$ & $0.25^{\mathrm{ijk}}$ & $15^{\text {hijk }}$ \\
\hline & 2007 & $0.087^{\mathrm{jklmnop}}$ & $422.6^{\circ}$ & $2^{\mathrm{bc}}$ & $33^{\mathrm{fg}}$ & $82.3^{\mathrm{jk}}$ & $146^{\mathrm{ef}}$ & $0.023^{\mathrm{k}}$ & $15^{\mathrm{hijk}}$ \\
\hline \multirow[t]{3}{*}{ Yaba-Clinic $^{\mathrm{D}}$} & 2008 & $0.150^{\mathrm{ijk}}$ & $394^{\mathrm{pq}}$ & $2^{\mathrm{bc}}$ & $32^{\mathrm{fgh}}$ & $79^{\mathrm{jk}}$ & $138^{\mathrm{ef}}$ & $5.35^{\mathrm{d}}$ & \\
\hline & 2012 & $0.083^{\mathrm{jklmnop}}$ & $383^{\mathrm{gr}}$ & $2.33^{\mathrm{ab}}$ & $34^{\mathrm{fg}}$ & $82.3^{\mathrm{jk}}$ & $142^{\mathrm{ef}}$ & $0.09^{\mathrm{jk}}$ & $14^{\mathrm{hijk}}$ \\
\hline & 2007 & $1.95^{\mathrm{a}}$ & $2465 \mathrm{a}$ & $1^{\mathrm{d}}$ & $407^{\mathrm{a}}$ & $1015^{\mathrm{a}}$ & $1447^{\mathrm{a}}$ & $0.5^{\mathrm{i}}$ & $105^{\mathrm{a}}$ \\
\hline \multirow[t]{3}{*}{ Wawara-COPROD ${ }^{\mathrm{D}}$} & 2008 & $1.95^{\mathrm{a}}$ & $2465 \mathrm{a}$ & $1^{\mathrm{d}}$ & $410^{\mathrm{a}}$ & $1015^{\mathrm{a}}$ & $1447.3^{\mathrm{a}}$ & $0.5^{\mathrm{i}}$ & $105^{\mathrm{a}}$ \\
\hline & 2012 & $1.65^{\mathrm{b}}$ & $2215 b$ & $1^{\mathrm{d}}$ & $405^{\mathrm{a}}$ & $1012^{\mathrm{a}}$ & $1446.3^{\mathrm{a}}$ & $0.4^{\mathrm{i}}$ & $100^{\mathrm{b}}$ \\
\hline & 2007 & $0.020^{\mathrm{op}}$ & $370.3^{\mathrm{rs}}$ & $1^{\mathrm{d}}$ & $32^{\mathrm{fgh}}$ & $80^{\mathrm{jk}}$ & $113.6^{\mathrm{ef}}$ & $0.02^{\mathrm{k}}$ & $9^{1}$ \\
\hline \multirow[t]{3}{*}{ Dioroum-PNGT ${ }^{\mathrm{MW}}$} & 2008 & $0.013^{\mathrm{op}}$ & $407.6^{\mathrm{p}}$ & $1^{\mathrm{d}}$ & $30^{\mathrm{fgh}}$ & $75^{\mathrm{k}}$ & $453^{\text {bcd }}$ & $0.3^{\mathrm{ijk}}$ & $11^{\mathrm{jkl}}$ \\
\hline & 2012 & $0.020^{\mathrm{op}}$ & $360^{\mathrm{s}}$ & $1^{\mathrm{d}}$ & $28^{\text {ghi }}$ & $81^{\mathrm{jk}}$ & $116^{\mathrm{ef}}$ & $0.02^{\mathrm{k}}$ & $10^{\mathrm{kl}}$ \\
\hline & 2007 & $0.020^{\mathrm{op}}$ & $71.3^{\mathrm{z}}$ & $1^{\mathrm{d}}$ & $11^{\mathrm{mn}}$ & $28^{\mathrm{p}}$ & $35.3^{\mathrm{f}}$ & $0.04^{\mathrm{k}}$ & $2^{\mathrm{m}}$ \\
\hline \multirow[t]{3}{*}{ Kassoum-Market $^{\mathrm{MW}}$} & 2008 & $0.323^{g}$ & $60^{\mathrm{z}}$ & $1^{\mathrm{d}}$ & $8^{\text {no }}$ & $20^{\mathrm{q}}$ & $27^{\mathrm{f}}$ & $0.2^{\mathrm{ijk}}$ & $2^{\mathrm{m}}$ \\
\hline & 2012 & $0.020^{\mathrm{op}}$ & $51^{z}$ & $1^{\mathrm{d}}$ & $15^{\operatorname{lm}}$ & $18^{\mathrm{qr}}$ & $25^{\mathrm{f}}$ & $0.08^{\mathrm{jk}}$ & $2^{\mathrm{m}}$ \\
\hline & 2007 & $0.110^{\mathrm{ijklmn}}$ & $467^{\mathrm{m}}$ & $1^{\mathrm{d}}$ & $35^{\mathrm{fg}}$ & $87.3^{\mathrm{j}}$ & $157.3^{\text {ef }}$ & $9.5^{\mathrm{b}}$ & $17^{\mathrm{hi}}$ \\
\hline \multirow[t]{3}{*}{ Yaba-Clinic $^{\mathrm{MW}}$} & 2008 & $0.113^{\mathrm{ijklmn}}$ & $468^{\mathrm{m}}$ & $1^{\mathrm{d}}$ & $35.66^{\mathrm{f}}$ & $87.3^{\mathrm{j}}$ & $157.6^{\mathrm{ef}}$ & $9.5^{\mathrm{b}}$ & $17^{\mathrm{hi}}$ \\
\hline & 2012 & $0.100^{\mathrm{jklmno}}$ & $442^{\mathrm{n}}$ & $1^{\mathrm{d}}$ & $32^{\mathrm{fgh}}$ & $85^{5^{\mathrm{jk}}}$ & $155^{\mathrm{ef}}$ & $0.1^{\mathrm{jk}}$ & $17^{\mathrm{hi}}$ \\
\hline & 2007 & $0.250^{\mathrm{h}}$ & $46^{z}$ & $2.66^{\mathrm{a}}$ & $4.16^{\circ}$ & $10^{\mathrm{qr}}$ & $21^{\mathrm{f}}$ & $0.08^{\mathrm{jk}}$ & $3^{\mathrm{m}}$ \\
\hline \multirow[t]{3}{*}{ Kouy-Mosque $^{\mathrm{TW}}$} & 2008 & $0.250^{\mathrm{h}}$ & $50^{\mathrm{z}}$ & $2^{\mathrm{bc}}$ & $3.1^{\circ}$ & $8^{\mathrm{r}}$ & $16^{\mathrm{f}}$ & $0.15^{\mathrm{jk}}$ & $3^{\mathrm{m}}$ \\
\hline & 2012 & $0.500^{\mathrm{d}}$ & $54^{\mathrm{z}}$ & $2^{\mathrm{bc}}$ & $5^{\circ}$ & $11^{\mathrm{qr}}$ & $22.6^{\mathrm{f}}$ & $0.32^{\mathrm{ijk}}$ & $2.3^{\mathrm{m}}$ \\
\hline & 2007 & $0.083^{\mathrm{jklmnop}}$ & $172^{\mathrm{ux}}$ & $1^{\mathrm{d}}$ & $19^{\mathrm{kl}}$ & $48^{\mathrm{mn}}$ & $64^{\mathrm{ef}}$ & $0.1^{\mathrm{jk}}$ & $4^{\mathrm{m}}$ \\
\hline \multirow[t]{2}{*}{ Sono-Centre ${ }^{\mathrm{TW}}$} & 2008 & $0.130^{\mathrm{ijklm}}$ & $179^{\mathrm{wux}}$ & $1^{\mathrm{d}}$ & $19^{\mathrm{kl}}$ & $47^{\mathrm{mno}}$ & $63^{\text {ef }}$ & $0.4^{\mathrm{ij}}$ & $4^{\mathrm{m}}$ \\
\hline & 2012 & $0.180^{\mathrm{i}}$ & $191^{\mathrm{wu}}$ & $1^{\mathrm{d}}$ & $18^{\mathrm{kl}}$ & $46^{\mathrm{mno}}$ & $62^{\mathrm{ef}}$ & $0.3^{\mathrm{ijk}}$ & $4^{\mathrm{m}}$ \\
\hline $\begin{array}{c}\text { Guideline values } \\
{[20-22]}\end{array}$ & - & $<350$ & 10 & - & - & - & $\leq 0.30$ & - & - \\
\hline
\end{tabular}

Means with a same letter within a column are not significantly different according to Newman Keuls' test $\mathrm{p}<0.05 .{ }^{\mathrm{D}} \mathrm{drilling} ;{ }^{\mathrm{MW}}$ modern well; ${ }^{\mathrm{TW}}$ traditional well.

effect of magnesium, calcium or hardness on cardiovascular mortality, the evidence is being debated and does not prove causality. There are insufficient data to suggest either minimum or maximum concentrations of minerals, as adequate intake will depend on a range of other factors. Therefore, no guideline values are proposed [20-22]. 
Out of the 15 water sources examined, 3 drillings (Di, Diouroum and Yaba) and one modern well (Yaba) provided water with Nitrates concentrations exceeding the WHO and USEPA guideline value $\left(50 \mathrm{mg} \mathrm{NO}_{3}^{-} / 1\right)[15$, 16]. The mean values for all the study periods were 123 , 740,113 , and $143 \mathrm{mg} \mathrm{NO}_{3}^{-} / 1$ for these water sources, respectively. The highest concentrations of nitrates were recorded in 2007 for the drilling of Diouroum and the modern well of Yaba (860 and $240 \mathrm{mg} / \mathrm{l}$, respectively), in 2008 for the drilling of Yaba (134 mg/l), and in 2012 for the drilling of Di (128 mg/l) (Table 2(a)). Nitrates concentration in drinking water is more focused because high level can be hazardous to infants. The nitrate itself is not a direct toxicant but is a health hazard because of its conversion to nitrite, which reacts with blood haemoglobin to cause methaemoglobinaemia. Hence, $50 \mathrm{mg}$ $\mathrm{NO}_{3}^{-} / 1$ nitrate is set as Guideline standard for nitrate in drinking water [20-22]. Concerning the study zone, concentrations higher than $100 \mathrm{mg} \mathrm{NO}_{3}^{-} / 1$ were observed by FAO [31] in the areas of Mouhoun and Sourou. Moreover, Nabayaogo [32] within the framework of a study on the impact of agricultural management on the water resources and the ecosystems of the Sourou valley, found nitrates contents of 2.7 to $37.2 \mathrm{mg} / \mathrm{l}$ for some wells and drillings located in Niassan village and on the riverside. These values reported by the author correspond approximately to the range of values recorded in this same village for drillings during the present study (11 to 18.3 $\mathrm{mg} / \mathrm{l})$. Dugué [33] concluded that the pollution risk of the aquifer by nitrates of agricultural origin is nearly zero in the Sourou valley (zone of Di). However, concentrations reaching $41 \mathrm{mg} \mathrm{NO}_{3}^{-} / 1$ were evidenced at the beginning of rainy season in water of the Sourou River, particularly downstream Di village, within the framework of this project and these concentrations could be due to the cultivation practices in line with the production of tomato and onions [17]. For the nitrates concentrations in groundwater exceeding sometimes at a large extent the WHO standard in the Sourou valley, investigations were performed to elucidate the origin [15]. Several tracks of contamination were thus evoked near the works, in relation with the anthropic activities: animal and human wild defecation, waste discharges, wastewaters rejections and so on. It is particularly the case at Diouroum village where the ground is strewn with excrements. In addition, specific organic matter deposits such as dunghills and composting areas can also generate rejections of nutrients. Latrines, although not very widespread in the Sourou villages can also punctually influence the quality of water (case of Yaba village), particularly in fractured zones where a fast contamination of the aquifer is possible. To these possible causes of nitrates pollution, the use of dynamite for digging on the groundwater quality was suggested since many explosives contain in their structure a nitrate radical which could remain in water after drilling. However, according to the authors, the precise diagnosis is not obvious to establish and it requires more in-depth investigation. Moreover, bacterial oxidation and fixation of nitrogen by plants can both produce nitrates [31]. Concerning nitrates originating from agricultural inputs, no connection between the fertilizers used and the contamination of groundwaters could be rigorously established, since most of the water supply points are far away from the hydro-agricultural perimeters. Moreover, samples collected from Niassan village, next to the irrigated perimeters presented nitrates concentrations lower than the guideline value for drinking water [20-22].

Throughout the study periods, mean potassium concentrations ranged from 0.2 - $132 \mathrm{mg} \mathrm{K}^{+} / 1$ (Table 2(a)). Rather high potassium concentrations were observed for $2(13 \%)$ of the water sources examined, namely Wawara (128 - $132 \mathrm{mg} / \mathrm{l})$ and Di $(55-120 \mathrm{mg} / \mathrm{l})$ drillings. As for nitrates, the variation in concentration, was related to the sampling period $(\mathrm{p}<0.0001)$, the highest values being observed in 2008 for these water sources (132 and 120 $\mathrm{mg} / \mathrm{l}$, respectively). Potassium is an essential element in humans and is seldom, if ever found in drinking water at levels that could be a concern for healthy humans. The recommended daily requirement is greater than $3000 \mathrm{mg}$ [20]. Potassium occurs widely in the environment, including all natural waters. Currently, there is no evidence that potassium levels in municipally treated drinking water, even water treated with potassium permanganate, are likely to pose any risk for the health of consumers [20]. Therefore, it is not considered necessary to establish a health-based guideline value for potassium in drinking water. Although potassium may cause some health effects in susceptible individuals, potassium intake from drinking water is well below the level at which adverse health effects may occur. Health concerns would be related to the consumption of drinking water treated by potassium-based water treatment (principally potassium chloride for regeneration of ion exchange water softeners), affecting only individuals in high-risk groups (i.e. individuals with kidney dysfunction or other diseases, such as heart disease, coronary artery disease, hypertension, diabetes, adrenal insufficiency, pre-existing hyperkalemia, people taking medications that interfere with normal potassium-dependent functions in the body, and older individuals or infants) [20].

For all the 15 water sources examined, the mean Sodium concentrations ranged from 0.5 to $19 \mathrm{mg} \mathrm{Na}{ }^{+} / 1$ (Table 2(a)). Although concentrations of sodium in potable water are typically less than $20 \mathrm{mg} / \mathrm{l}$, they can greatly exceed this in some countries. No firm conclusions can be drawn concerning the possible association between sodium in drinking water and the occurrence of hypertension. Therefore, no health-based guideline value 
is proposed. However, concentrations in excess of 200 $\mathrm{mg} / \mathrm{l}$ may give rise to unacceptable taste [20].

The mean Magnesium and Calcium concentrations ranged from $2-105 \mathrm{mg} \mathrm{Mg}^{+} / 1$ and $4-410 \mathrm{mg} \mathrm{Ca}^{2+} / 1$ (Table 2(b)). Both parameters varied significantly with the water source $(\mathrm{p}<0.01)$ and the sampling period $(\mathrm{p}<$ 0.05) (Table 1(b)). Garzon and Eisenberg [34] first showed that there is a large variation in mineral contents of commercially available bottled drinking waters. The magnesium content of bottled water available in North America ranged from 1 to $120 \mathrm{mg} / \mathrm{l}$, and the calcium content ranged from 1 to $240 \mathrm{mg} / \mathrm{l}$, whereas concentration in bottled waters that are commercially available in Europe ranged from 0 to $546 \mathrm{mg} / \mathrm{l}$ for calcium and from 1 to $126 \mathrm{mg} / \mathrm{l}$ for magnesium. Comprehensive follow-up studies [35-37] on a wide range of commercially available bottled waters suggested that the mineral levels varied tremendously in bottled waters within countries and around the world. As summarized by WHO [38] from the above sources, magnesium and calcium concentrations found in water ranged from $0-29 \mathrm{mg} \mathrm{Mg}^{2+} / 1$ and $2-83$ $\mathrm{mg} \mathrm{Ca}^{2+} / 1$ for surface water sources, $2-48 \mathrm{mg} \mathrm{Mg}^{2+} / 1$ and $26-85 \mathrm{mg} \mathrm{Ca}^{2+} / 1$ for groundwater sources, $1-130 \mathrm{mg}$ $\mathrm{Mg}^{2+} / 1$ and $3-310 \mathrm{mg} \mathrm{Ca}^{2+} / 1$ for Mineral water. Therefore, out of the 15 water sources studied, only the drilling of Wawara (7\% of the water sources) with concentrations of $100-105 \mathrm{mg} \mathrm{Mg}^{2+} / 1$ and $405-410 \mathrm{mg} \mathrm{Ca}^{2+} / 1$ can be classified as highly mineralized, while the other water sources $(93 \%)$ crossed the values found for groundwater sources. Magnesium and Calcium are naturally occurring in surface or groundwater from erosion and weathering of soils, minerals or ores. There is no evidence of adverse health effects from calcium or magnesium in drinking water; both ions contribute to water hardness. Therefore, guideline values are not proposed [20,22,39].

Total Iron in water samples ranged from $0.02-9.8 \mathrm{mg}$ $\mathrm{Fe} / 1$ (Table 2(b)). These concentrations varied significantly with the sampling site and the sampling period as well ( $p<0.0001$; Tables 1(b) and 2(b)). Concentrations over the WHO and USEPA Guideline value $(\leq 0.3 \mathrm{mg}$ $\mathrm{Fe} / \mathrm{l}$ ) were found in water samples from the modern well of Yaba and the drillings of Diouroum, Niassan-clinic, Sono, Yaba and Kouy $(6.3,3.3,2.3,2.2,1.8$, and $1 \mathrm{mg}$ $\mathrm{Fe} / \mathrm{l}$, respectively). Iron is one of the most abundant metals in Earth's crust. It is found in natural fresh waters at levels ranging from 0.5 to $50 \mathrm{mg} / 1$ [20]. Toxic effects have resulted from the ingestion of large quantities of iron, but there is no evidence to indicate that concentrations of iron commonly present in drinking water constitute any hazard to human health; hence, a maximum acceptable concentration has not been set. However; at concentrations above $0.3 \mathrm{mg} / \mathrm{l}$ (drinking water standard), iron can stain laundry and plumbing fixtures and produce undesirable tastes in beverages. The precipitation of ex- cessive iron impacts an objectionable reddish-brown color to water and may also promote the growth of certain microorganisms (i.e. Iron-Reducing Bacteria), leading to the deposition of a slimy coating in water distribution pipes $[20,31,39]$.

Ammonium concentrations in the water samples ranged from $0.002-1.95 \mathrm{mg} \mathrm{NH} \mathrm{NH}_{4}^{+} / 1$ (Table 2(b)). Concentrations recorded in the water samples varied significantly with the sampling site and the sampling period as well ( $p<0.0001$, Table 1(b)). The term ammonia includes the non-ionized $\left(\mathrm{NH}_{3}\right)$ and ionized $\left(\mathrm{NH}_{4}{ }^{+}\right)$species. Ammonia in the environment originates from metabolic, agricultural and industrial processes and from disinfection with chloramine. Natural levels in groundwater and surface water are usually below $0.2 \mathrm{mg} / \mathrm{l}$. Anaerobic groundwaters may contain up to $3 \mathrm{mg} / \mathrm{l}$. Intensive rearing of farm animals can give rise to much higher levels in surface water. Ammonia in water is an indicator of possible bacterial, sewage and animal waste pollution. It represents a major component of the metabolism of mammals. Exposure from environmental sources is insignificant in comparison with endogenous synthesis of ammonia. Toxicological effects are observed only at exposures above about $200 \mathrm{mg} / \mathrm{kg}$ body weight. Ammonia in drinking-water is not of immediate health relevance, and therefore no health-based guideline value is proposed $[20,22]$. However, ammonia can compromise disinfecttion efficiency, result in nitrite formation in distribution systems; it can also cause the failure of filters for the removal of manganese, taste and odor problems [20].

Mean Sulfates concentrations in the water samples ranged from 1.5 - $1301.66 \mathrm{mg} \mathrm{SO} \mathrm{SO}_{4}^{2-} / 1$ (Table 2(a)). Concentrations were significantly related to the sampling site and the sampling period as well ( $\mathrm{p}<0.0001$, Table 1(a)). Sulfates occur naturally in numerous minerals and are used commercially, principally in the chemical industry. The highest levels usually occur in groundwater and are from natural sources. In general, the average daily intake of sulfates from drinking water, air and food is approximately $500 \mathrm{mg}$, food being the major source. However, in areas with drinking water supplies containing high levels of sulfates, drinking water may constitute the principal source of intake [20-22]. No health-based guideline is proposed for sulfate. However, because of the gastrointestinal effects (diarrhea or dehydration) resulting from ingestion of drinking water containing high sulfates levels, it is recommended that health authorities be notified of sources of drinking water that contain sulfate concentrations in excess of $500 \mathrm{mg} / \mathrm{l}$. The presence of sulfates in drinking water may also cause noticeable taste over 250 $\mathrm{mg} / \mathrm{l}$ (threshold taste) and may contribute to the corrosion of distribution systems [20-22]. From the data recorded, out of the 15 water sources examined, only the drilling of Wawara $(1175-1301.66 \mathrm{mg} / \mathrm{l})$ appears to be of health 
concern regarding the defined standard $(\leq 500 \mathrm{mg} / \mathrm{l})$. The high sulfates levels leading to noticeable taste may partially explain the rejection by the village populations of this drilling for their alimentation as revealed our field investigations.

The mean Arsenic concentrations recorded for all the water sources studied were in the range of $1-2.66 \mu \mathrm{g} / \mathrm{l}$ and crossed the guideline value for the maximum acceptable concentration of Arsenic in drinking water [20-22]. Arsenic is found widely in Earth's crust in oxidation states of $-3,0,+3$ and +5 , often as sulfides or metal arsenides or arsenates. In water, it is mostly present as arsenate $(+5)$, but in anaerobic conditions, it is likely to be present as arsenite $(+3)$. Levels in natural waters generally range between 1 and $2 \mu \mathrm{g} / \mathrm{l}$, although concentrations may be elevated (up to $12 \mathrm{mg} / \mathrm{l}$ ) in areas containing natural sources [20]. Arsenic has not been demonstrated to be essential in humans. The acute toxicity of arsenic compounds in humans is predominantly a function of their rate of removal from the body. Arsine is considered to be the most toxic form, followed by the arsenites, the arsenates and organic arsenic compounds. Acute arsenic intoxication associated with the ingestion of well water containing very high concentrations $(21.0 \mathrm{mg} / \mathrm{l})$ of arsenic has been reported [20]. Numerous epidemiological studies have examined the risk of cancers associated with arsenic ingestion through drinking water. The International Program on Chemical Safety (IPCS) concluded that long-term exposure to arsenic in drinking-water is causally related to increased risks of cancer in the skin, lungs, bladder and kidney, as well as other skin changes, such as hyperkeratosis and pigmentation changes. In view of the practical difficulties in removing arsenic from drinking water, particularly from small supplies, and the practical quantification limit for arsenic $(1-10 \mu \mathrm{g} / \mathrm{l})$, the guideline value of $10 \mu \mathrm{g} / \mathrm{l}$ is retained as a goal [20].

\subsection{Water Microbiology}

The results of the microbiological examination of the water sources samples showing Escherichia coli and fecal Coliforms cells count throughout the sampling periods are presented in Tables 3 and 4. All the drillings were exempt of fecal pollution and crossed the recommended guideline values [20-22]. By contrast, water from wells appeared uniformly polluted with concentrations exceeding sometimes $10^{3}$ and $10^{4} \mathrm{CFU} / 100 \mathrm{ml}$ for $E$. coli and fecal Coliforms, respectively (Table 4). For the latters, water pollution by both indicators was significantly $(\mathrm{p}<0.0001)$ related to the water source location and the sampling period as well (Table 3). Since wells are not protected from environmental contaminations (Figures 3(c) and (d)), they may receive several depositions with regard to the absence of latrines in rural area, animals frequentation and the atmospheric conditions leading to water microbial pollution $[15,17]$. These potential sources or amplifying factors of microbial pollution may vary with the sampling source location and/or the sampling period as evidenced the data recorded (Table 3).

Field investigations showed a preference of wells as source of water for the alimentation of populations in the Sourou Valley, and that appeared mainly related to the high water hardness or sulfates content (noticeable taste) of water provided by some drillings (i.e. Wawara), poverty and cultural considerations. Therefore, these populations are exposed to health risks [20-22] as underlined previous investigations in the zone [40-42].

\subsection{Drinking Water Quality}

Physico-chemical and microbiological parameters were analyzed to identify the physical status, impurities, other dissolved substances and microorganisms that affect water used for domestic purposes in the Sourou valley.

From the 15 water sources examined, water from 4 (26\%) drillings was of health concern in view of nitrates concentration (Di, Yaba and Diouroum), sulfates content (Wawara) and the guideline values for these parameters, respectively $[11,12,20-22]$. The 6 other drillings (40\%) can be classified as safe sources for drinking water with regards to the physico-chemical and microbiological qualities, guideline standards and health risks.

All water samples from wells $(33 \%$ of water sources studied) appeared uniformly polluted from fecal contaminations with concentrations of $E$. coli and fecal Coliforms largely over the guideline values [11,12,20-22].

Overall, $9(60 \%)$ of the water sources examined do not provide safe water for populations alimentation. As a consequence of such unhygienic water quality, waterborne diseases have proven to be the biggest health threat worldwide and they contribute between $70 \%-80 \%$ of health problems in developing countries $[1,28]$. These diseases continue to be a major cause of human mortality and morbidity. Diarrheal diseases remain a leading cause of illness and death in the developing world which alone causes 2.2 million of the 3.4 million water-related deaths per year, $90 \%$ of these deaths involving children less than five years $[1,20,28]$.

As revealed studies $[17,40]$ and the present investigations, populations in the Sourou valley are preferentially getting drinking water from wells and even do from the surface water of the Sourou River, although presenting unsafe quality. What could be the reasons undergoing such situation in the Sourou valley?

Field investigations (group discussions and interviews) were performed to elucidate the situation and to propose some issues for improving safe drinking water access for 
Table 3. Variance of bacterial fecal pollution indicators in water from drillings and wells with regard to sampling site and sampling period.

\begin{tabular}{cccccc}
\hline \multirow{2}{*}{ Source of variation } & df & \multicolumn{2}{c}{ Escherichia coli $(\mathrm{CFU} / 100 \mathrm{ml})$} & \multicolumn{2}{c}{ Fecal Coliforms (CFU/100 ml) } \\
\cline { 3 - 6 } & & MS & $\mathrm{p}$ & $\mathrm{MS}$ & $\mathrm{p}$ \\
\hline Site & 14 & 2414336.67 & $<0.0001^{* *}$ & 68372495.25 & $<0.0001^{* *}$ \\
Period & 2 & 3171524.12 & $<0.0001^{* *}$ & 76018114.69 & $<0.0001^{* *}$ \\
Site*Period & 28 & 1440370.22 & $<0.000^{1 * *}$ & 33793213.73 & $<0.000^{1 * *}$ \\
\hline
\end{tabular}

${ }^{\mathrm{MS}}$ mean square; ${ }^{* *}$ significant $\mathrm{p}<0.01$.

Table 4. Means* concentrations of $E$. coli and fecal Coliforms in water from drillings and wells during the dry season in the Sourou valley in 2007, 2008, and 2012 ("mean of 3replicates).

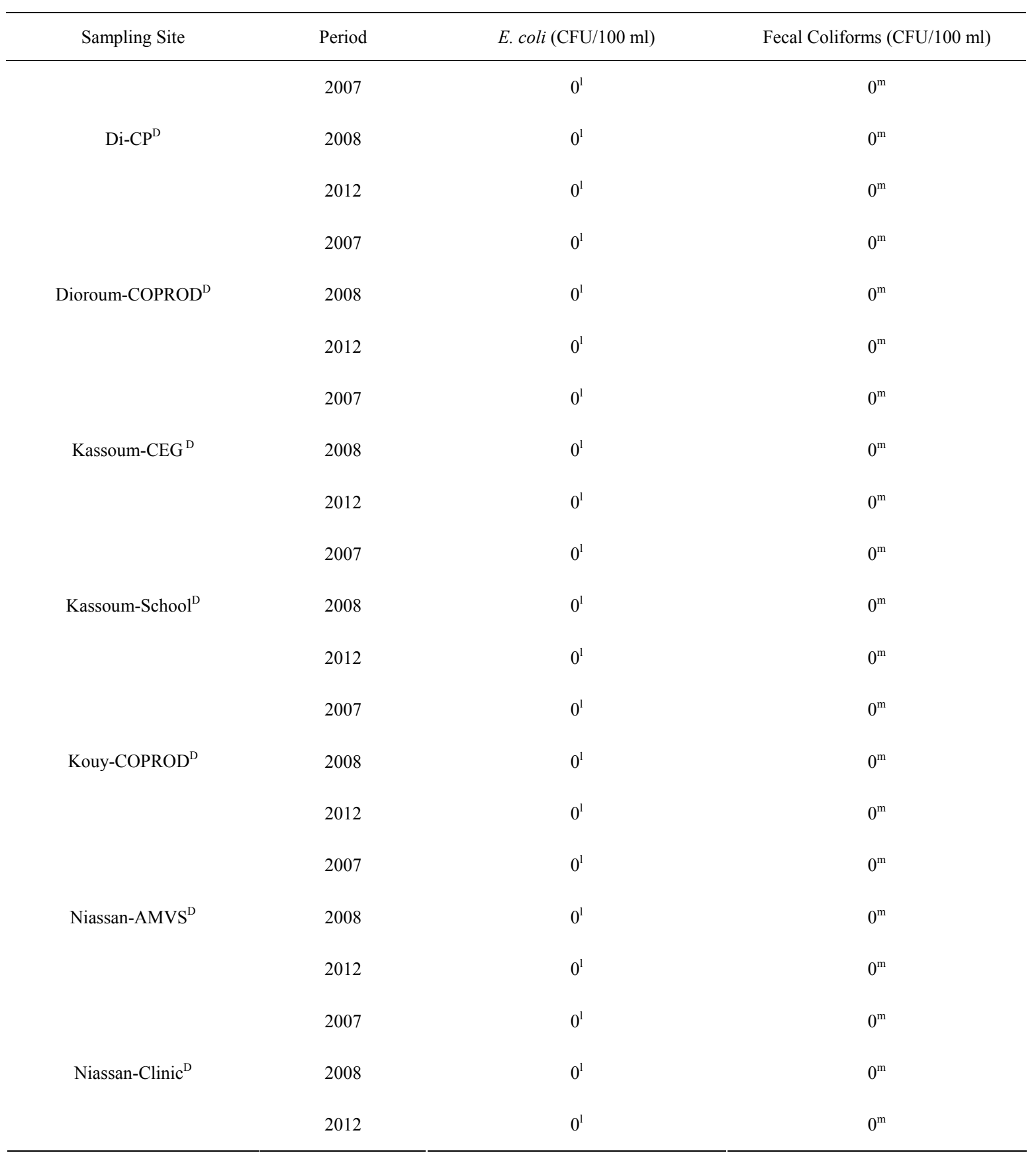




\section{Continued}

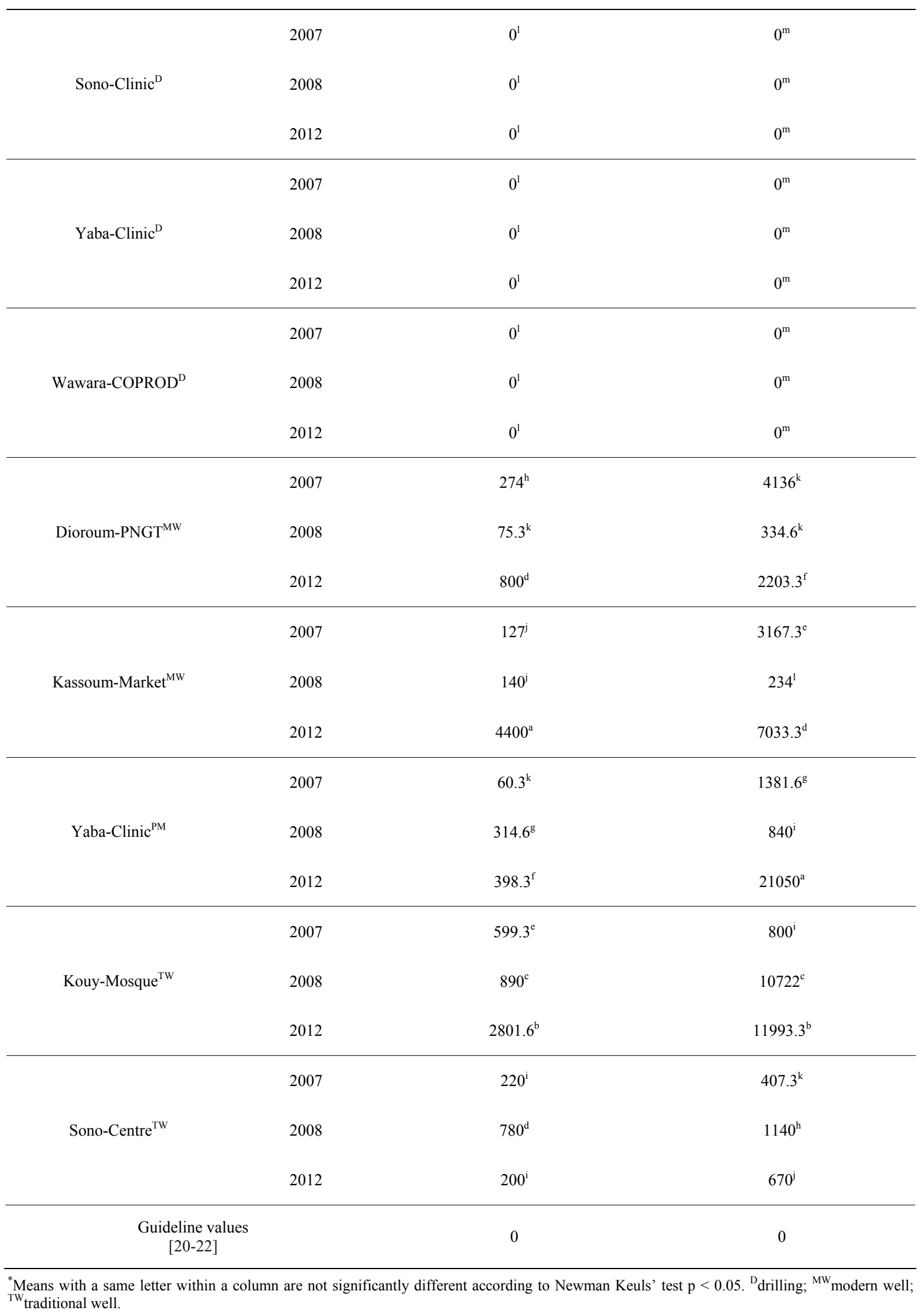


populations in the Sourou valley.

\subsection{Problematic of Safe Drinking Water Access in the Sourou Valley}

Although according to the United Nations Development Program, Burkina Faso already reached the MDG for the access to safe drinking water [13], the situation is nottherefore satisfactory, in particular in rural environment as underlined the Ministry in charge of water management [14].

The improvement these years in the access to safe drinking water in the country, focused mainly on the quantitative aspect through the reinforcement of the infrastructures of water supply [13]. However, beyond the quantitative aspect, it is also advisable to take consideration on the quality of water consumed by populations.

In the Sourou valley, populations fed on water from drillings, modern and traditional wells, and from the surface water of the Sourou River as well (Figures 2(a)-(e)) $[17,40]$. As revealed the data recorded, if some drillings ( $60 \%$ of those examined) provide safe drinking water, in contrast, other drillings (40\%) deliver water with either high nitrates [10] or sulfates concentrations over the guideline values [11,12,20-22]. Moreover, water from all the wells examined $(100 \%)$ was microbiologically polluted (Table 4).

Field investigations revealed some factors contributing to the non-achievement in the access to safe drinking water for populations in the Sourou valley.

\subsubsection{The Non-Regular Control of Water Quality}

If in urban environment, distributed water is the object of regular control, it is not the case in rural environment where the indicators of drinking water quality are missing due to the lack of analytical data. For all the drillings examined in the study, no report attesting the quality of water provided by the hydraulic realization prior to populations' utilization was found. This situation illustrates clearly a lack of control during the realization of drillings.

As a consequence, some drillings are providing unsafe drinking water to populations. Moreover, although the APIWRM recommended the installation of national networks to monitor water quality, water uses, water requests and health risks, such operational structures remain to be created.

In line with this gap and to meet the recommendation of APIWRM [7,18], the present study intends to improve the knowledge related to the quality of drinking water resources in the Sourou basin [15-19].

\subsubsection{The Lack of Self-Management of Infrastructures for Drinking Water Supply}

During field investigations, several drillings were found broken down and not repaired. According to Ouédraogo
[43], this apparent indifference in the water resource management, could be related to the fact that water is free and in addition, the hydraulic realization is a donation of government or NGO. Therefore, populations are just waiting for the donators to repair.

Such situation was well illustrated during the campaign of drillings restoration by the COPROD, one action of the Sourou river contract. Within this framework, a hundred broken down drillings were repaired but some were broken down again after a few months and were never been repaired by the populations [16]. This negative perception of self-water resource management by populations could be in line with a lack of education and sensitization. These gaps can be overcome, since primary school through the sensitization for an efficient integrated water resources management [16]. Unfortunately, such pedagogic activities are relatively scarce in Burkina Faso, although the intention is underlined in the APIWRM action plan [7].

\subsubsection{Socio-Cultural Considerations}

During samples collection, one remarkable observation was that water sources are mainly frequented by women and children (Figures 2(a) and (d)). This situation is related to the socio-cultural context, for which these fractions of the population are mainly in charge of household chores [19].

Moreover, wells appeared much more frequented than drillings for drinking water gathering in some villages (Figure 2(c)). As revealed investigations from water users, collecting water from wells gives opportunity to exchange on social and current events than drillings can afford [23].

For populations of villages located on the Sourou River, only the surface water is used for their alimentation (Figure 2(e)). All efforts from local authorities encouraging these populations to leave the unsafe environment were unsuccessful. This attitude in line with traditional believes leads these populations to consume unsafe water exposing them to waterborne diseases as revealed previous investigations in the zone [17,40-42].

\subsubsection{The Lack of Hygiene and Basic Sanitation}

In developing countries, the lack of hygiene and basic sanitation is still among the main factors limiting the access to safe drinking water for rural populations, despite efforts at international and national levels to provide water facilities. In addition to the factors limiting safe drinking water access evoqued above, the absence of disinfecting methods of drinking water in household should be also evoqued [44-47]. It has also been indicated that even do for water distribution networks, the duration of water storage in pipe, temperature variations and pipe materials can lead to bacterial proliferation in 
water collected in household from water pump $[48,49]$.

Although we didn't examine the quality of drinking water in households, field observations supported a poor microbial quality of water from drillings in households as revealed the aspects of water collecting and storing materials (Figure 2(a)) and the method of water transportation to household (Figure 2(f)). The conesquences of such practices and attitudes on the quality of drinking water were highlighted in previous studies $[44,50]$.

\subsection{Some Issues to Improve Drinking Water Quality and Access in the Sourou Valley}

In line with the limited safe drinking water access for populations in the Sourou valley, the OXFAM Belgique in collaboration with the Water Unit of Liège University and the Rotary Club Burkina Faso undertook to equip school classes with a local material-based technology (Figure 4) for preserving drinking water safety for school children. Over improving drinking water quality, these actions contributed to the fight against poverty through a financial support to local associations in charge of setting the technology design. Although the analysis of water is- sued from the disposal showed a rather good quality at microbiological level (data not shown), some improvement of the technology is needed to obtain a safe drinking water in line with the guideline standards [11,12,20-22].

Investigations using local plant material (grains of Moringa oleifera) to improve the quality of some surface waters and water from wells which feed the populations in the Sourou valley are currently performed [47]. These investigations, the ones in RDC Congo [45,46] and the outputs from previous studies in Burkina Faso [44,50] may help improving the quality of drinking water for rural populations in Sub-Saharan developing countries.

Beyond these direct actions intending to improve the quality of drinking water, it is also advisable within the framework of an integrated approach, to develop preventive measures at land management and soil occupation nearby water sources, and at hygiene and basic sanitation levels as well.

Therefore, the construction of protection zones around drinking water sources, the promotion of hygiene-based rules and the installation of bottom-tight latrines could help improving drinking water quality for rural populations in the Sourou valley and in developing countries.

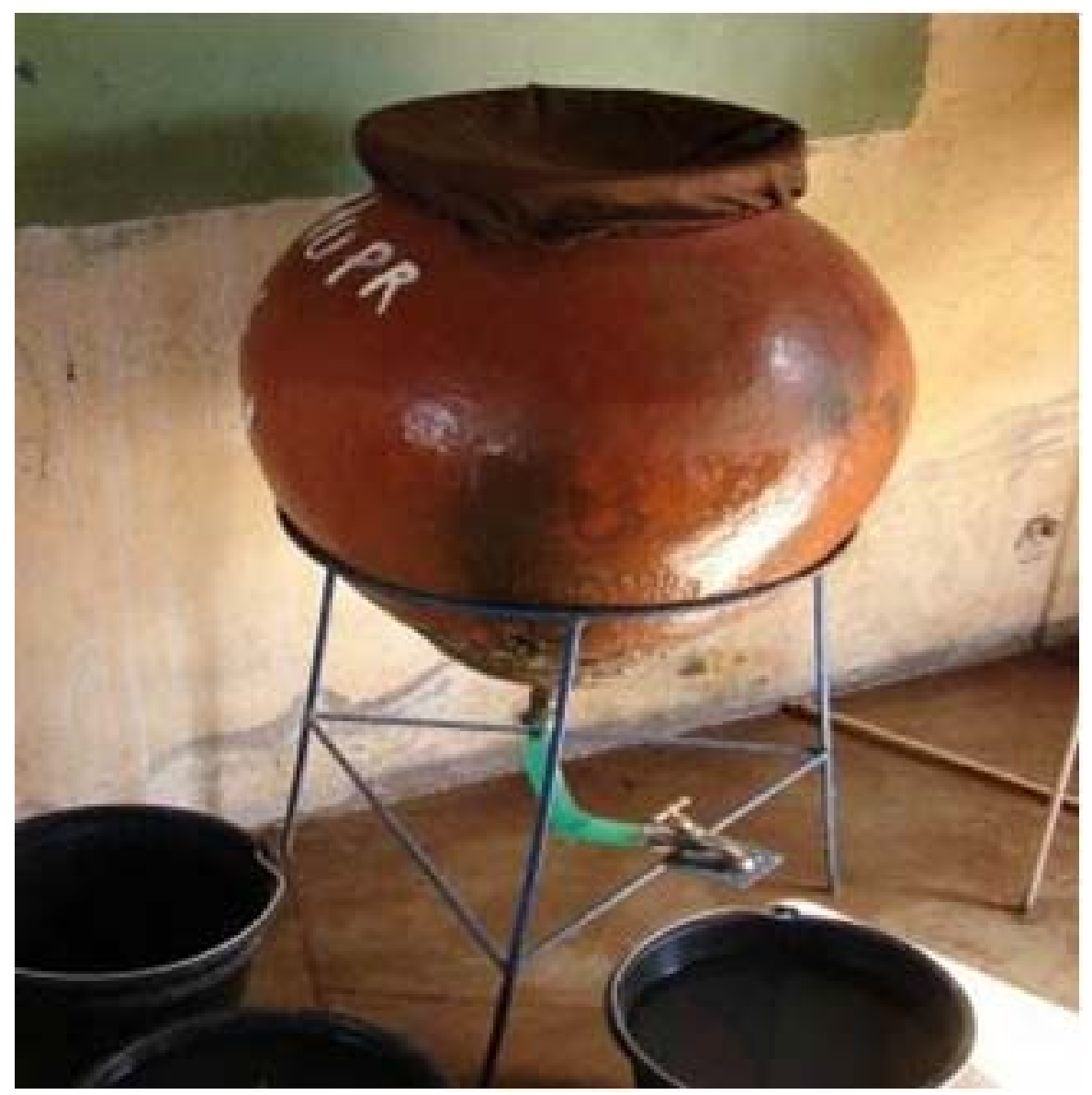

Figure 4. Technology based on local material experienced in schools of the Sourou valley for the preservation of safe drinking water quality. 


\section{Conclusions and Prospects}

Access to safe drinking water for rural populations in the Sourou valley is still facing the optimization of water resources management. The lack of an efficient network of water quality survey and a self-management of drinking water sources facilities, the inertia of mentalities and traditional believes appear the main constraints governing this problematic in the Sourou valley. To overcome these constraints, information and sensitization actions on water resources management and on water and health relationships are required. This approach involves pedagogic aspect and should therefore be performed in collaboration with school teachers, focusing children and women the main actors at the center of the problematic.

In addition, scientific expertise, through improving knowledge on water resources quality and providing appropriate methods for water disinfection in households could help solving the problematic. Moreover, an integrated approach which associates water and land manaments could allow setting preventive measures for the protection of drinking water sources in the Sourou valley.

Overall, these proposed issues for improving the access to safe drinking water for populations must be provided to the institutions in charge of the water policy and to the local collectivities, which within the framework of the decentralization, were seen entrusted the responsibility for the natural resources management. It is also advisable that local participating structures as the Local Water Committees (LWC) catch these problems in order to improve the water services in response to the populations needs with respect to the national and international standards of drinking water.

\section{Acknowledgements}

The authors would like to express profound gratitude to the Walloon Region of Belgique, University of Liège, CNRST/IRSS, INERA-Di, COPROD, LNAE and Tougan Hospital for providing financial support, laboratory and other facilities.

\section{REFERENCES}

[1] World Health Organization, "Guidelines for Drinking Water Quality," 3rd Edition, World Health Organization, Geneva, 2008.

[2] United Nations Development Programme, "Burkina Faso: Access to Safe Drinking Water Has Improved a Lot," 2010.

[3] United Nations Environment Program, UNEP, "The Problematic of Water in the Democratic Republic of Congo," Technical Report, 2011.

[4] K. G. R. Kouam, H. G. Mpakam, S. A. Ndonwy, S. L. D. Bopda and G. E. Ekodeck, "Integrated Management of Water Resources and Millennium Development Goals for the Development of Africa: Case study of Cameroun," Vertigo, Vol. 7, No. 2, 2006, pp. 1-9.

[5] F. Julien, "Water Control and Sustainable Development in West Africa: A Need of a Regional Cooperation on the Trans-Boundaries Hydrologic Systems," Vertigo, Vol. 7, No. 2, 2006, pp. 1-18.

[6] D. J. H. Phillips, M. Daoudy, S. C. McCaffrey and A. J. R. Öjendal, "Trans-Boundary Water Cooperation as a Tool for Conflict Prevention and Broader Benefit Sharing," Swedish Ministry of Foreign Affairs, Stockholm, 2006, p. 249.

[7] Ministry of Agriculture, "National Action Plan for Integrated Water Resources Management (APIWRM)," $\mathrm{Hy}$ draulics and Fishing Resources, Ouagadougou, 2003, p. 62.

[8] Ministry of Water, "Leaflet of the Hydrogeological Map of Burkina Faso, Scale 1:500,000, Sheet Bobo-Dioulasso," Direction of Hydraulics Resources Inventory, 1993, p. 40.

[9] Ministry of Agriculture, "Conception Plan and Implementation of the National Water Information System," Direction of Hydraulics Resources Inventory, Hydraulics and Fishing Resources, 2004, p. 135.

[10] Ministry of Agriculture, "National Program of Drinking Water and Sanitation Supply for the 2015 year issue (NPDWSS2015)," Direction of Hydraulics Resources Inventory, Hydraulics and Fishing Resources, 2006, p. 43.

[11] Ministry of Water and Environment, "Decree n 2001-185/ PRES/PM/MEE, Fixing the Standards of Pollutants in Air, Water and Soil," 7 May 2001.

[12] World Health Organization, "Guidelines for Drinking Water Quality," Third Edition, Geneva, 2004.

[13] United Nations, United Nations Development Programme, "Access to Safe Drinking Water," 2010.

[14] Ministry of Agriculture, "IWRM 2010 and 2011 Perspectives," Hydraulics and Fishing Resources, Ouagadougou, 2011, p. 29.

[15] F. Rosillon, B. Savadogo, A. Kaboré, H. Bado-Sama and D. Dianou, "Attempts to Answer on the Origin of the High Nitrates Concentrations in Groundwaters of the Sourou Valley in Burkina Faso," Journal of Water Resource and Protection, Vol. 4, No. 8, 2012, pp. 663-673. doi:10.4236/jwarp.2012.48077

[16] F. Rosillon, B. Savadogo, A. Kaboré, H. Bado-Sama and D. Dianou, "Estimation of the Nitrates Contents in Waters by Using Reagent Strips: An Environment Education Exercise in the Sourou Valley in Burkina Faso," Vertigo, Vol. 12, No. 2.

[17] D. Dianou, B. Savadogo, D. Zongo, T. Zougouri, J. N. Poda, H. Bado and F. Rosillon, "Surface Waters Quality of the Sourou Valley: The Case of Mouhoun, Sourou, Debe and Gana Rivers in Burkina Faso," International Journal of Biological and Chemical Sciences, Vol. 5, No. 4, 2011, pp. 1571-1589.

[18] Ministry of Agriculture, "IWRM 2010 and 2011 Perspectives," Hydraulics and Fishing Resources, Ouagadougou, 2011, p. 29. 
[19] F. Rosillon, P. Vander Borght and H. Bado-Sama, "River Contract in Wallonia (Belgium) and Its Application for Water Management in the Sourou Valley (Burkina Faso)," Water Sciences and Technology, Vol. 52, No. 9, 2005, pp. 85-93.

[20] World Health Organization, "Guidelines for Drinking Water Quality," Fourth Edition, World Health Organization, Geneva, 2011.

[21] United States Environmental Protection Agency "Drinking Water Standards and Health Advisories," 2012, p. 12.

[22] Health Canada, "Guidelines for Canadian Drinking Water Quality Summary Table," Water, Air and Climate Change Bureau, Healthy Environments and Consumer Safety Branch, Health Canada, Ottawa, p. 22.

[23] H. Bado-Sama and F. Rosillon, "River Contract of Sourou, Outcome of the Actions Program 2003-2008," Report COPROD/ULG," 2009, p. 76.

[24] Direction of Hydraulics Resources Inventory, Ministry of Water and Burkina Faso, "Leaflet of the Hydrogeological Map of Burkina Faso Scale 1:500,000 Sheet Bobo-Dioulasso," 1993, p. 40.

[25] American Public Health Association, American Water Works Association and World Peace Choral Festival, "Standard Methods for the Examination of Water and Wastewater," 20th Edition, by American Public Health Association, American Water Works Association and Water Pollution Control Federation, Washington, 1998.

[26] J. Rodier, "Water Analysis: Natural Waters, Wastewaters, Sea Water,” Ed. Dunod, Paris, 2005, p. 1384.

[27] G. I. Oyhakilome, A. F. Aiyesanmi and F. C. Akharaiyi, "Water Quality Assessment of the Owena Multi-Purpose Dam, Ondo State, Southwestern Nigeria," Journal of Environmental Protection, Vol. 3, No. 1, 2012, pp. 14-25. doi:10.4236/jep.2012.31003

[28] L. J. Bina, T. Prasai, A. Singh and K. D. Yami, “Assessment of Drinking Water Quality of Madhyapur-Thimi and Study of Antibiotic Sensitivity Against Bacterial Isolates," Nepal Journal of Science and Technology, Vol. 10, 2009, pp. 167-172.

[29] I. D. McKelvie, "In Situ Measurement of Physicochemical Water Quality Parameters," In: F. R. Burden, D. Donnert, T. Godish and I. D. McKelvie, Eds., Environmental Monitoring Handbook, McGraw-Hill Co., Boston, 2004, pp. 3.1-3.21.

[30] Ireland Environmental Protection Agency, "Parameters of Water Quality: Interpretation and Standards," Environmental Protection Agency, Johnstown, 2001, p. 133.

[31] FAO, "Aquastat, Water and Agriculture FAO Information System: Burkina Faso,” 2005.

[32] D. Nabayaogo, "Agricultural Activity in the Sourou Valley: Impact on Water Resources and Ecosystems," Memory, University of Ouagadougou, 2006, p. 42.

[33] P. Dugué, "Environmental Assessment Study and Development of Sustainable Production Systems in Projects to Support Food Production in Mali and Burkina Faso," Foundation for Agriculture and Rural Life in the World, CIRAD, 2009, p. 97.
[34] P. Garzon and M.J. Eisenberg, "Variation in the Mineral Content of Commercially Available Bottled Waters: Implications for Health and Disease," The American Journal of Medicine, Vol. 105, No. 2, 1998, pp. 125-130. doi:10.1016/S0002-9343(98)00189-2

[35] A. Von Wiesenberger, "The Pocket Guide to Bottled Water," Contemporary Books, Chicago.

[36] A. Azoulay, P. Garzon, and M.J. Eisenberg, "Comparison of the Mineral Content of Tap Water and Bottled Waters," Journal of General Internal Medicine, Vol. 16, No. 3, 2001, pp. 168-175. doi:10.1111/j.1525-1497.2001.04189.x

[37] I. Rosborg, B. Nihlgard, L. Gerhardsson, M. L. Gernersson, R. Ohlin and T. Olsson, "Concentrations of Inorganic Elements in Bottled Waters on the Swedish Market," Environmental Geochemistry and Health, Vol. 27, No. 3, 2005, pp. 217-227. doi:10.1007/s10653-004-1612-8

[38] World Health Organization, "Calcium and Magnesium in drinking water: Public Health Significance, World Health Organization, Geneva, 2009.

[39] European Union's Drinking Water Standards, “Council Directive 98/83/EC on the Quality of Water Intended for Human Consumption," Geneva, 1998.

[40] D. Dianou, J. N. Poda, L. G. Savadogo, H. Sorgho, S. P. Wango and B. Sondo, "Intestinal parasites in the zone of the hydro-agricultural complex of Sourou in Burkina," Vertigo, Vol. 5, No. 2, 2004, pp. 1-8.

[41] J. N. Poda, J. R. Mwanga, D. Dianou, A. Garba, F. C. Ouédraogo, D. Zongo and K. B. Sondo, "Parasite Infections that Undermine the Pole of Development in Burkina Faso: Case Study of Schistosomiasis and Geohelminthiasis in the Hydro-Agricultural Complex of Sourou," Vertigo, Vol. 7, No. 2, 2006, pp. 1-7.

[42] A. Koukounari, A. F. Gabrielli, S. Toure, E. Bosque-Oliva, Y. Zhang, B. Sellin, C. A. Donnelly, A. Fenwick and J. P. Webster, "Schistosoma haematobium Infection and Morbidity before and after Large-Scale Administration of Praziquantel in Burkina Faso," Journal of Infectious Diseases, Vol. 196, No. 5, 2007, pp. 659-669. doi:10.1086/520515

[43] B. Ouedraogo, "Water Management and Environmental Education in the Pedagogic Documents in Burkina Faso," Vertigo, 25 December 2003. http://vertigo.revues.org/1968

[44] D. Dianou, J. N. Poda, L. Thiombiano and H. Sorgho, "Evaluation of Contamination Tracks and Some Disinfecting Methods of Households' Drinking Water in Rural Area: Case Study of Thion village in Burkina Faso," Annals University of Ouagadougou, Vol. C, No. 2, 2004, pp. 168-181.

[45] M. Sunda, F. Rosillon and K. M. Taba, "Contribution to Water Disinfection through Photosensitization with Plant Extracts," Environment Journal of Water Quality, Vol. 39, No. 2, 2008, pp. 199-209. doi:10.1051/water/2008006

[46] M. Sunda, "Contribution to Water Disinfection through Photosensitization with Plant Extracts," Ph.D. Dissertation, University of Liège, Faculty of Sciences, 2012.

[47] A. Kaboré, B. Savadogo, F. Rosillon, A. S. Traoré and D. 
Dianou, "Optimization of the Effectiveness of Moringa Oleifera Seeds in the Treatment of Drinking Water in Sub-Saharan Africa: Case Study of Waters of Burkina Faso," Annals University of Ouagadougou, Vol. 9, 2011, pp. 880-889.

[48] K. Lautenschlager, N. Boon, Y. Wang, T. Egli and F. Hammes, "Overnight Stagnation of Drinking Water in Household Taps Induces Microbial Growth and Changes in Community Composition," Water Research, Vol. 44, No. 17, 2010, pp. 4868-4877. doi:10.1016/j.watres.2010.07.032

[49] A. S. Patrick, A. Roland, C. Ananil and S. B. Dampare, "Nutrient-Induced Growth of Coliform and HPC Bacteria in Drinking Water Pipes," Journal of Environmental Protection, Vol. 3, No. 6, 2012, pp. 508-517 doi:10.4236/jep.2012.36061

[50] D. Dianou, J. N. Poda, H. Sorgho and L. Thiombiano, "Quality of Households Drinking Water at Thion, Blédougou and Kangoura in Burkina Faso," South Sciences and Technologies, Vol. 9, 2002, pp. 25-33. 